\title{
A Novel Memory Filtering Design for Semi-Markovian Jump Time-Delay Systems
}

\author{
Yanling Wei, Member, IEEE, Jianbin Qiu ${ }^{(0)}$, Senior Member, IEEE, \\ Hamid Reza Karimi ${ }^{\circledR}$, Senior Member, IEEE, and Wenqiang Ji
}

\begin{abstract}
This paper investigates the problem of delay-dependent $\mathscr{H}_{\infty}$ memory filtering for continuous-time semi-Markovian jump linear systems (MJLSs) with time-varying delay in an input-output framework. Differing from the constant transition rates (TRs) in the conventional MJLSs, the TRs of the semi-MJLSs depend on the random sojourn-time and are thus with time-varying characteristics. By utilizing a two-term approximation for the terms with time-varying delay, it is first shown that the filtering error system (FES) can be reformulated into a feedback interconnection form and the stability and performance analysis problem of the FES can be recast as the scaled small gain (SSG) problem of an interconnected system. Then, based on a semi-Markovian Lyapunov-Krasovskii formulation of SSG condition combined with projection lemma, the $\mathscr{H}_{\infty}$ filter synthesis for the underlying semi-MJLSs is formulated in terms of linear matrix inequalities. Finally, simulation studies are provided to evaluate the effectiveness and superiority of the proposed design method.
\end{abstract}

Index Terms-Cumulative distribution function (CDF), $\mathscr{H}_{\infty}$ filtering, scaled small gain (SSG) theorem, semi-Markovian jump systems, time-varying delay.

\section{INTRODUCTION}

$\mathbf{J}$ UMP linear systems (JLSs) are usually defined as a family of linear systems with randomly jumping parameters [1], [2]. It has been widely verified that JLSs are powerful to model dynamical systems suffered from random failures or structural changes, such as networked control systems, fault-prone power systems and economics systems, and so on [3]-[9]. It is known that JLSs are intrinsically with finitestate operation modes, and the mode transitions are determined by a stochastic process [10], [11]. Essentially, the stochastic process relies on the duration $h$ between two successive

Manuscript received May 24, 2017; revised September 2, 2017; accepted September 18, 2017. Date of publication October 19, 2017; date of current version November 15, 2018. This work was supported in part by the National Natural Science Foundation of China under Grant 61503091 and Grant 61522306, and in part by the State Key Laboratory of Robotics and Systems, Harbin Institute of Technology under Grant SKLRS-2017-KF-02. This paper was recommended by Associate Editor Z. Wang. (Corresponding author: Jianbin Qiu.)

Y. Wei, J. Qiu, and W. Ji are with the Research Institute of Intelligent Control and Systems, Harbin Institute of Technology, Harbin 150080 , China, and also with the State Key Laboratory of Robotics and Systems, Harbin Institute of Technology, Harbin 150080, China (e-mail: jianbinqiu@gmail.com).

H. R. Karimi is with the Department of Mechanical Engineering, Politecnico di Milano, 20156 Milan, Italy.

Color versions of one or more of the figures in this paper are available online. jumps, which is also termed as sojourn-time. It is worth mentioning that the sojourn-time $h$ is some random variable with a probability distribution $F$. When $F$ serves as an exponential distribution, and the transition rates (TRs) $\lambda_{i j}(h)=\lambda_{i j}$ are thus time-invariant as the memoryless feature of the exponential distribution. The latter indicates that the switchings are merely determined by the latest state. Such situations are commonly experienced in Markovian JLSs (MJLSs) [12]-[20].

In practice, however, it is not always the case that the practical systems can be restricted with the memoryless feature. More general, the TRs $\lambda_{i j}(h)$ could be sojourn-timedependent rather than fixed. A continuous stochastic process with sojourn-time obeying nonexponential distribution is usually defined as a semi-Markov process. In such case, a JLS that operates with a semi-Markov process is addressed as a semiMJLS [21]-[26]. It is obvious that the traditional MJLSs are a particular type of semi-MJLSs that can be utilized to model and describe a broader class of practical stochastic systems. Hence, it is potential in both theoretical advances and engineering applications to study the analysis and synthesis issues of semi-MJLSs, which partially inspires this paper.

Additionally, it has been shown that time-delays are frequently experienced in different practical control systems, and the existence of time-delays is an origin of instability, oscillation, and performance degradation. Thus, there have been many results involving delay-dependent and delay-independent types on the analysis and design for time-delay systems and it has been revealed that the delay-dependent criteria generally reflect the reality better [27]-[29]. It is worth mentioning that the reported delay-dependent criteria are basically proposed by the direct Lyapunov method. More recently, inspired by the robust control theory [30], another promising but indirect framework to deal with time-delays is the IO method, which primarily depends on the model reformulation and scaled small gain (SSG) theorem [31]. In retrospect, this approach was initially introduced in the robust stability analysis field, then it was employed to cope with linear systems with constant delay in [32] and further to time-varying delay cases in [33] and [34]. Inevitably, time-delays also exist in MJLSs, and therefore to further reduce conservativeness, it is of much importance to investigate the analysis and design of semi-MJLSs with timevarying delay by virtue of the IO method, which is the main purpose of this investigation.

As the dual of control problem, the topic of state estimation prevails in signal processing and control engineering 
applications. Accordingly, various protocols for estimation and filtering design have been developed such as the Kalman filtering approach [35], the $\mathscr{H}_{2}$ approach [36], the $\mathscr{L}_{2}-\mathscr{L}_{\infty}$ approach [37], and the $\mathscr{H}_{\infty}$ approach [38], [39]. Specifically, the $\mathscr{H}_{\infty}$ filtering has attracted considerable attention due to its advantages over Kalman filtering that $\mathscr{H}_{\infty}$ filtering is of insensitiveness to the information of the external noise signals and more robustness to parametric uncertainties [37][39]. However, notice that most obtained results on $\mathscr{H}_{\infty}$ filter design for time-delay systems were developed based on a memoryless filter [38], [39]. For conservativeness reduction purpose, a memory filter, which contains the time-delay information in the filter model, seems more reasonable and powerful [40], [41]. Nevertheless, it is worth pointing out that few results on $\mathscr{H}_{\infty}$ memory filtering have been presented for semiMJLSs with time-varying delay, which is another inspiration for this paper.

According to the issues mentioned above, in this paper, we will tackle the delay-dependent $\mathscr{H}_{\infty}$ memory filtering problem for continuous-time semi-MJLSs with time-varying delay via an IO approach. Concretely, with a two-term approximation (TTA) to the state-delay, the underlying system will be initially converted into a feedback interconnection (FI) formulation, which involves a forward subsystem with two constant state-delays and a feedback one with normbounded uncertainties. Then, according to a semi-Markovian Lyapunov-Krasovskii (MLK) format of stochastic SSG condition together with projection lemma, the $\mathscr{H}_{\infty}$ performance analysis and filter synthesis will be proposed. In particular, both full-order and reduced-order filter parameters can be computed in a convex optimization frame. Simulation studies will be finally preformed to reveal the effectiveness and advantages of the developed method.

Notations: The notations utilized are standard. $\mathbb{R}_{+}$and $\mathbb{N}_{+}$ denote, respectively, the set of non-negative real numbers and the set of non-negative integers; $\operatorname{Sym}\{A\}$ refers to $A+A^{\top}$; $\mathcal{E}[\cdot]$ represents the mathematical expectation; $\|\cdot\|$ refers to the Euclidean norm of a vector or its induced norm of a matrix; and signals that are square integrable over $[0, \infty)$ is represented by $L_{2}[0, \infty)$ with the norm $\|\cdot\|_{2}$.

\section{PROBLEM DESCRIPTIONS}

This paper considers the subsequent continuous-time semiMJLSs with time-varying state-delay

$$
\left\{\begin{array}{c}
\dot{x}(t)=A(r(t)) x(t)+A_{d}(r(t)) x(t-d(t)) \\
\quad+B(r(t)) w(t) \\
y(t)=C(r(t)) x(t)+C_{d}(r(t)) x(t-d(t)) \\
\quad+D_{1}(r(t)) w(t) \\
z(t)=L(r(t)) x(t)+L_{d}(r(t)) x(t-d(t)) \\
\quad+D_{2}(r(t)) w(t) \\
x(t)=\phi_{t}, \quad t \in\left[-d_{2}, 0\right]
\end{array}\right.
$$

where $x(t) \in \mathbf{R}^{n_{x}}$ refers to the state vector, $w(t) \in \mathbf{R}^{n_{w}}$ the disturbance input belonging to $L_{2}[0, \infty), y(t) \in \mathbf{R}^{n_{y}}$ the measurement output, and $z(t) \in \mathbf{R}^{n_{z}}$ the signal to be estimated. In addition, $d(t)$ refers to a time-varying delay satisfying $0 \leq d_{1} \leq d(t) \leq d_{2}<\infty$ and $\dot{d}(t) \leq \mu<\infty$, where $\left\{d_{1}, d_{2}\right\} \in \mathbb{R}_{+}$denotes the lower and upper bounds of timedelay, respectively. In (1), $\phi_{t}$ provides the initial condition of states during $\left[-d_{2}, 0\right] ;\{r(t), h\}_{t \geq 0}:=\left\{r_{n}, h_{n}\right\}_{n \in \mathbb{N}_{\geq 1}}$ is characterized by a continuous-time semi-Markov process that takes the values in a finite set $\mathcal{I}:=\{1, \ldots, N\}$, where $\left\{r_{n}\right\}_{n \in \mathbb{Z}_{+}}$is the index of system mode at the $n$th transition, taking values in $\mathcal{I}$, and $\left\{h_{n}\right\}_{n \in \mathbb{Z}_{+}}$is the sojourn-time of mode $r_{n-1}$ between the $(n-1)$ th transition and $n$th transition, taking values in $\mathbb{R}_{+}$[24]-[26]. The evolution of semi-Markov process is governed by the following transition probabilities:

$$
\left\{\begin{array}{c}
\operatorname{Pr}\left\{r_{n+1}=j, h_{n+1} \leq h+\delta \mid r_{n}=i, h_{n+1}>h\right\} \\
=\lambda_{i j}(h) \delta+o(\delta), \quad i \neq j \\
\operatorname{Pr}\left\{r_{n+1}=j, h_{n+1}>h+\delta \mid r_{n}=i, h_{n+1}>h\right\} \\
=1+\lambda_{i i}(h) \delta+o(\delta), \quad i=j
\end{array}\right.
$$

where $h$ denotes the sojourn-time that indicates the time duration between two successive mode transitions; $o(\delta)$ is the little- $o$ notation defined as $\lim _{\delta \rightarrow 0}(o(\delta) / \delta)=0$, and $\lambda_{i j}(h) \geq 0$, for $j \neq i$, denotes the TR from mode $i$ at time $t$ to mode $j$ at time $t+\delta$, and $\lambda_{i i}(h)=-\sum_{j=1, j \neq i}^{N} \lambda_{i j}(h)$. In particular, $\left(A_{i}, A_{d i}, B_{i}, C_{i}, C_{d i}, D_{1 i}, L_{i}, L_{d i}, D_{2 i}\right)$ refers to the system matrices of the $i$ th mode.

For the stochastic stability and $\mathscr{H}_{\infty}$ disturbance attenuation level, we introduce the subsequent definitions.

Definition 1 [25]: The system in (1) with semi-Markovian jump parameters is stochastically stable (SS) if there exists a finite positive constant $T\left(x_{0}, r_{0}\right)$ to make the subsequent inequality hold for any initial condition $\left(x_{0}, r_{0}\right)$

$$
\mathcal{E}\left[\int_{0}^{\infty}\|x(t)\|^{2} \mathrm{~d} t \mid\left(x_{0}, r_{0}\right)\right] \leq T\left(x_{0}, r_{0}\right) .
$$

Definition 2 [25]: System (1) achieves the stochastic stability with an $\mathscr{H}_{\infty}$ performance $\gamma$ if the following two requirements are met.

1) System (1) with $w(t) \equiv 0$ is $\mathrm{SS}$ in the sense of Definition 1.

2) In the context of zero initial conditions and any nonzero $w(t) \in L_{2}[0, \infty)$, the inequality $\mathcal{E}\left\{\int_{0}^{\infty}\|z(t)\|^{2} \mathrm{~d} t\right\} \leq$ $\gamma^{2} \int_{0}^{\infty}\|w(t)\|^{2} \mathrm{~d} t$ holds.

Throughout this paper, the following assumption is made. Assumption 1: System (1) is SS.

In accordance with the semi-MJLS in (1), we aim at synthesizing the subsequent mode-dependent memory filter

$$
\left\{\begin{array}{r}
\dot{\hat{x}}(t)=A_{f 0 i} \hat{x}(t)+\frac{1}{2} A_{f 1 i} \hat{x}\left(t-d_{1}\right) \\
+\frac{1}{2} A_{f 2 i} \hat{x}\left(t-d_{2}\right)+B_{f i} y(t) \\
\hat{z}(t)=C_{f 0 i} \hat{x}(t)+\frac{1}{2} C_{f 1 i} \hat{x}\left(t-d_{1}\right) \\
+\frac{1}{2} C_{f 2 i} \hat{x}\left(t-d_{2}\right)+D_{f i} y(t)
\end{array}\right.
$$

where $\hat{x}(t) \in \mathbf{R}^{n_{f}}\left(n_{f} \leq n_{x}\right)$ denotes the filter state; $\hat{z}(t) \in \mathbf{R}^{n_{z}}$ refers to the estimation of $z(t)$; and $\left\{A_{f 0 i}, A_{f 1 i}, A_{f 2 i}\right\} \in \mathbf{R}^{n_{f} \times n_{f}}$, $B_{f i} \in \mathbf{R}^{n_{f} \times n_{y}},\left\{C_{f 0 i}, C_{f 1 i}, C_{f 2 i}\right\} \in \mathbf{R}^{n_{z} \times n_{f}}$, and $D_{f i} \in \mathbf{R}^{n_{z} \times n_{y}}$, $\forall i \in \mathcal{I}$, are filter parameters to be designed. Notice that $n_{f}=$ $n_{x}$ for the full-order filter and $n_{f}<n_{x}$ for the reduced-order filter, and when $A_{f 1 i}=A_{f 2 i}=\mathbf{0}$ and $C_{f 1 i}=C_{f 2 i}=\mathbf{0}$, then (3) recovers to a traditional memoryless filter.

Defining the augmented state vector $\bar{x}(t)$ := $\left[\begin{array}{ll}x^{\top}(t) & \hat{x}^{\top}(t)\end{array}\right]^{\top}$, and $\bar{z}(t):=z(t)-\hat{z}(t)$, we can attain 
the subsequent filtering error system (FES)

$$
\left\{\begin{array}{c}
\dot{\bar{x}}(t)=\bar{A}_{0 i} \bar{x}(t)+\frac{1}{2} \bar{A}_{d 1 i} \bar{x}\left(t-d_{1}\right)+\frac{1}{2} \bar{A}_{d 2 i} \bar{x}\left(t-d_{2}\right) \\
\quad+\bar{A}_{d i} \bar{x}(t-d(t))+\bar{B}_{i} w(t) \\
\bar{z}(t)=\bar{L}_{0 i} \bar{x}(t)+\frac{1}{2} \bar{L}_{d 1 i} \bar{x}\left(t-d_{1}\right)+\frac{1}{2} \bar{L}_{d 2 i} \bar{x}\left(t-d_{2}\right) \\
+\bar{L}_{d i} \bar{x}(t-d(t))+\bar{D}_{i} w(t) \\
\bar{x}(t)=\left[\begin{array}{ll}
\phi_{t}^{\top} & \mathbf{0}
\end{array}\right]^{\top}, t \in\left[-d_{2}, 0\right]
\end{array}\right.
$$

where

$$
\left\{\begin{array}{l}
\bar{A}_{0 i}:=\left[\begin{array}{cc}
A_{i} & \mathbf{0} \\
B_{f i} C_{i} & A_{f 0 i}
\end{array}\right], \bar{A}_{d 1 i}:=\left[\begin{array}{cc}
\mathbf{0} & \mathbf{0} \\
\mathbf{0} & A_{f 1 i}
\end{array}\right] \\
\bar{A}_{d 2 i}:=\left[\begin{array}{cc}
\mathbf{0} & \mathbf{0} \\
\mathbf{0} & A_{f 2 i}
\end{array}\right], \bar{A}_{d i}:=\left[\begin{array}{cc}
A_{d i} & \mathbf{0} \\
B_{f i} C_{d i} & \mathbf{0}
\end{array}\right] \\
\bar{B}_{i}:=\left[\begin{array}{ll}
B_{i}^{\top} & D_{1 i}^{\top} B_{f i}^{\top}
\end{array}\right] \\
\bar{L}_{0 i}:=\left[\begin{array}{ll}
L_{i}-D_{f i} C_{i} & -C_{f 0 i}
\end{array}\right] \\
\bar{L}_{d 1 i}:=\left[\begin{array}{ll}
\mathbf{0} & -C_{f 1 i}
\end{array}\right], \bar{L}_{d 2 i}:=\left[\begin{array}{ll}
\mathbf{0} & -C_{f 2 i}
\end{array}\right] \\
\bar{L}_{d i}:=\left[\begin{array}{ll}
L_{d i}-D_{f i} C_{d i} & \mathbf{0}
\end{array}\right], \bar{D}_{i}:=D_{2 i}-D_{f i} D_{1 i} .
\end{array}\right.
$$

The purpose of this paper is to synthesize a memory filter to guarantee the stochastic stability with an $\mathscr{H}_{\infty}$ performance $\gamma$ for the FES (4) via an IO approach. More specifically, by performing a TTA to the state-delay, the original semiMJLS is transformed into a new IO interconnected format. Such framework reduces the stability analysis of the original time-delay systems to the analysis of FI systems with nominal constant time-delay part but with additional inputs and outputs. Indeed, the reformulation underscores the role that the SSG-type results may play in stability analysis of timedelay systems. In the sequel, we first present some concepts on the SSG theorem.

It is worth mentioning that the small gain theorem for deterministic systems has been extensively utilized in the systems and control community [33], [34]. Here, we will develop its counterpart version for semi-Markovian jump stochastic systems.

Definition 3: A mapping containing semi-Markovian jumps $\mathcal{L}: \xi(t) \rightarrow \eta(t)$ is IO SS, if there exists a non-negative constant $\gamma$ such that

$$
\|\eta\|_{\mathcal{E}_{2}} \leq \gamma\|\xi\|_{\mathcal{E}_{2}}
$$

for all $\xi$. Moreover, the gain of $\mathcal{L}$ is defined as $\gamma(\mathcal{L}):=$ $\sup _{\xi \neq 0}\left[\left(\|\eta\|_{\mathcal{E}_{2}}\right) /\left(\|\xi\|_{\mathcal{E}_{2}}\right)\right]$.

Consider an interconnected stochastic system composed by two subsystems

$$
\mathcal{L}_{1}: \eta_{1}(t)=G \xi_{1}(t), \quad \mathcal{L}_{2}: \eta_{2}(t)=\Delta \xi_{2}(t)
$$

which is depicted in Fig. 1, where the forward subsystem $\mathcal{L}_{1}$ is known with operator $G$ mapping $\xi_{1}$ to $\eta_{1}$, and the feedback one $\mathcal{L}_{2}$ is unknown with operator $\Delta$ mapping $\xi_{2}$ to $\eta_{2}$. Then we will derive the following stochastic small gain theorem.

Lemma 1 (Stochastic Small Gain Theorem): Consider the interconnected semi-MJLS (7). Then, the system is IO SS for all the subsystems $G$ and $\Delta$ satisfying

$$
\begin{aligned}
& \left\|G \xi_{1}\right\|_{\mathcal{E}_{2}} \leq k_{1}\left\|\xi_{1}\right\|_{\mathcal{E}_{2}}, k_{1}>0 \\
& \left\|\Delta \xi_{2}\right\|_{\mathcal{E}_{2}} \leq k_{2}\left\|\xi_{2}\right\|_{\mathcal{E}_{2}}, k_{2}>0
\end{aligned}
$$

if $k_{1} k_{2}<1$.

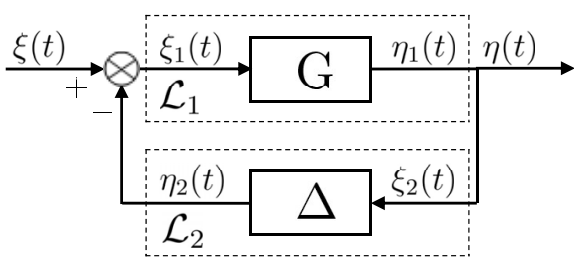

Fig. 1. Interconnected system composed by two subsystems.

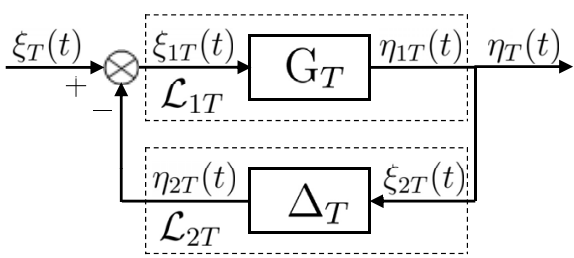

Fig. 2. Transformed system consisting of two subsystems.

Proof: Based on the condition (8), one has

$\|\eta\|_{\mathcal{E}_{2}} \leq k_{1}\left\|\xi_{1}\right\|_{\mathcal{E}_{2}}=k_{1}\|\xi-\Delta \eta\|_{\mathcal{E}_{2}} \leq k_{1}\left(\|\xi\|_{\mathcal{E}_{2}}+k_{2}\|\eta\|_{\mathcal{E}_{2}}\right)$.

Obviously with $k_{1} k_{2}<1$, we have

$$
\|\eta\|_{\mathcal{E}_{2}} \leq \frac{k_{1}}{1-k_{1} k_{2}}\|\xi\|_{\mathcal{E}_{2}}
$$

which indicates that the interconnected system in (7) is IO SS based on Definition 3. This completes the proof.

Thus, Lemma 1 (stochastic small gain theorem) provides a sufficient condition for the robustly stochastic stability of the FI system constructed by $\mathcal{L}_{1}$ and $\mathcal{L}_{2}$ in (7).

As a direct benefit of the stochastic small gain theorem, one can further obtain the following lemma.

Lemma 2: Consider the interconnected semi-MJLS (7). Then, the system is IO SS for all the subsystems $G$ satisfying

$$
\gamma(G)=\|G\|_{\infty}<1
$$

if $\|\Delta\|_{\infty} \leq 1$.

The proof of Lemma 2 can be readily carried out by following the similar lines of Lemma 1 .

Furthermore, we can obtain a stronger result by scaling. For any real invertible matrix $\left\{T_{\xi}, T_{\eta}\right\}$, let

$$
\begin{aligned}
& \xi_{1 T}:=T_{\xi} \xi_{1}, \quad \eta_{1 T}:=T_{\eta} \eta_{1}, \quad \xi_{2 T}:=T_{\eta} \xi_{2} \\
& \eta_{2 T}:=T_{\xi} \eta_{2}, \quad \xi_{T}:=T_{\xi} \xi, \quad \eta_{T}:=T_{\eta} \eta .
\end{aligned}
$$

Then the system (7) can be written as

$$
\begin{aligned}
& \mathcal{L}_{1 T}: \eta_{1 T}(t)=T_{\eta} G T_{\xi}^{-1} \xi_{1 T}(t):=G_{T} \xi_{1 T}(t) \\
& \mathcal{L}_{2 T}: \eta_{2 T}(t)=T_{\xi} \Delta T_{\eta}^{-1} \xi_{2 T}(t):=\Delta_{T} \xi_{2 T}(t) .
\end{aligned}
$$

This alternative expression amounts to redrawing the interconnection of Fig. 1 as in Fig. 2.

By the variable transformation in (12), we thus have

$$
\gamma\left(G_{T}\right)=\gamma\left(T_{\eta} G T_{\xi}^{-1}\right)=\sup _{\xi_{1} \neq 0} \frac{\left\|T_{\eta} \eta_{1}\right\|_{\mathcal{E}_{2}}}{\left\|T_{\xi} \xi_{1}\right\|_{\mathcal{E}_{2}}}
$$

and $\gamma\left(\Delta_{T}\right)=\gamma\left(T_{\xi} \Delta T_{\eta}^{-1}\right)$ can be obtained in a similar form. When $T_{\xi}$ and $T_{\eta}$ are the identity matrices, $\gamma\left(G_{T}\right)$ and $\gamma\left(\Delta_{T}\right)$ 
reduce to $\gamma(G)$ and $\gamma(\Delta)$, respectively. Then we have the following lemma.

Lemma 3 (Stochastic SSG Theorem): Consider (7) and suppose that $\mathcal{L}_{1}$ is IO SS. The FI system constituted by $\mathcal{L}_{1}$ and $\mathcal{L}_{2}$ is IO SS if $\left\|T_{\eta} G T_{\xi}^{-1}\right\|_{\infty}<1$ is satisfied for some matrices $\left\{T_{\xi}, T_{\eta}\right\} \in \mathcal{T}$ with $\mathcal{T}:=\left\{\left\{T_{\eta}, T_{\xi}\right\} \in \mathbf{R}^{\eta \times \eta} \times\right.$ $\mathbf{R}^{\xi \times \xi}: T_{\eta}, T_{\xi}$ nonsingular; $\left.\left\|T_{\xi} \Delta T_{\eta}^{-1}\right\|_{\infty} \leq 1\right\}$.

Proof: By considering the conditions $\left\|T_{\eta} G T_{\xi}^{-1}\right\|_{\infty}<1$ and $\left\|T_{\xi} \Delta T_{\eta}^{-1}\right\|_{\infty} \leq 1$, one achieves

$$
\gamma\left(G_{T}\right)<1, \gamma\left(\Delta_{T}\right) \leq 1
$$

According to Lemma 2, it is evident that the interconnected system $F_{T}=\operatorname{feedback}\left(G_{T}, \Delta_{T}\right)$ consisting of two semiMJLSs $\mathcal{L}_{1 T}: \xi_{1 T} \rightarrow \eta_{1 T}$ and $\mathcal{L}_{2 T}: \xi_{2 T} \rightarrow \eta_{2 T}$ is IO SS. On the other hand, from the variable transformation (12), we have

$$
\eta=F \xi=T_{\eta}^{-1} F_{T} T_{\xi} \xi,\|\eta\|_{\mathcal{E}_{2}} \leq\left\|T_{\eta}^{-1}\right\|_{2}\left\|F_{T} T_{\xi} \xi\right\|_{\mathcal{E}_{2}}
$$

which, together with the IO stochastic stability of $F_{T}$, leads to

$$
\begin{aligned}
\|\eta\|_{\mathcal{E}_{2}} & \leq\left\|T_{\eta}^{-1}\right\|_{2} \gamma\left(F_{T}\right)\left\|T_{\xi} \xi\right\|_{\mathcal{E}_{2}} \\
& \leq\left\|T_{\eta}^{-1}\right\|_{2} \gamma\left(F_{T}\right)\left\|T_{\xi}\right\|_{2}\|\xi\|_{\mathcal{E}_{2}} .
\end{aligned}
$$

The latter means that the interconnected system $F=$ feedback $(G, \Delta)$ is IO SS based on Definition 3, and thus the proof is completed.

\section{MAIN RESULTS}

In this section, we first reformulate the FES (4) into an FI of two subsystems as in (7) and analyze the stochastic SSG of the forward subsystem. By a semi-MLK format of stochastic SSG condition, a new bounded real lemma will be presented for system (4). Then, by virtue of a linearization technique together with projection lemma, the $\mathscr{H}_{\infty}$ filter synthesis is developed.

\section{A. Model Reformulation}

Following the idea of [33] and [34], taking the estimation of the time-delay state $\bar{x}(t-d(t))$ by the term $1 / 2\left[\bar{x}\left(t-d_{1}\right)+\right.$ $\left.\bar{x}\left(t-d_{2}\right)\right]$ achieves the approximation error:

$$
\begin{aligned}
\bar{\eta}_{d}(t) & =\bar{x}(t-d(t))-\frac{1}{2}\left[\bar{x}\left(t-d_{1}\right)+\bar{x}\left(t-d_{2}\right)\right] \\
& =\frac{1}{2} \int_{-d_{2}}^{-d(t)} \dot{\bar{x}}(t+\theta) \mathrm{d} \theta-\frac{1}{2} \int_{-d(t)}^{-d_{1}} \dot{\bar{x}}(t+\theta) \mathrm{d} \theta \\
& :=\frac{1}{2} \int_{-d_{2}}^{-d_{1}} \rho(\theta) \xi_{d}(t+\theta) \mathrm{d} \theta
\end{aligned}
$$

where $\xi_{d}(t):=\dot{\bar{x}}(t)$ and $\rho(\theta):= \begin{cases}1, & \text { when } \theta \leq-d(t) \\ -1, & \text { when } \theta>-d(t) .\end{cases}$

Performing the normalization of $\bar{\eta}_{d}(t)$ in (18) by multiplying $2 / d$ with $d=d_{2}-d_{1}$, (18) can be re-expressed with the form

$$
\eta_{d}(t):=\Delta_{d}(t) \xi_{d}(t)=\frac{1}{d} \int_{-d_{2}}^{-d_{1}} \rho(\theta) \xi_{d}(t+\theta) \mathrm{d} \theta
$$

where $\eta_{d}(t):=(2 / d) \bar{\eta}_{d}(t)$, and the operator $\Delta_{d}: \xi_{d} \mapsto \eta_{d}$, which involves the uncertainties pulled out from $\bar{x}(t-d(t))$ in the original time-delay system (4).
Substitution (19) into (4) leads to the subsequent FI system in an equivalent manner

$$
\begin{gathered}
\left(\mathcal{L}_{d 1}\right):\left\{\begin{array}{l}
\dot{\bar{x}}(t)=\bar{A}_{0 i} \bar{x}(t)+\frac{1}{2} \bar{A}_{1 i} \bar{x}\left(t-d_{1}\right) \\
\quad+\frac{1}{2} \bar{A}_{2 i} \bar{x}\left(t-d_{2}\right)+\frac{d}{2} \bar{A}_{d i} \eta_{d}(t)+\bar{B}_{i} w(t) \\
\bar{z}(t)=\bar{L}_{0 i} \bar{x}(t)+\frac{1}{2} \bar{L}_{1 i} \bar{x}\left(t-d_{1}\right) \\
\quad+\frac{1}{2} \bar{L}_{2 i} \bar{x}\left(t-d_{2}\right)+\frac{d}{2} \bar{L}_{d i} \eta_{d}(t)+\bar{D}_{i} w(t) \\
\xi_{d}(t)=\overline{\bar{x}}(t)
\end{array}\right. \\
\left(\mathcal{L}_{d 2}\right): \eta_{d}(t)=\Delta_{d}(t) \xi_{d}(t) \\
\bar{x}(t)=\left[\begin{array}{ll}
\phi_{t}^{\top} \quad \mathbf{0}
\end{array}\right]^{\top}, t \in\left[-d_{2}, 0\right]
\end{gathered}
$$

where

$$
\left\{\begin{array}{l}
\bar{A}_{0 i}:=\left[\begin{array}{cc}
A_{i} & \mathbf{0} \\
B_{f i} C_{i} & A_{f 0 i}
\end{array}\right], \bar{A}_{1 i}:=\left[\begin{array}{cc}
A_{d i} & \mathbf{0} \\
B_{f i} C_{d i} & A_{f 1 i}
\end{array}\right] \\
\bar{A}_{2 i}:=\left[\begin{array}{cc}
A_{d i} & \mathbf{0} \\
B_{f i} C_{d i} & A_{f 2 i}
\end{array}\right], \bar{A}_{d i}:=\left[\begin{array}{cc}
A_{d i} & \mathbf{0} \\
B_{f i} C_{d i} & \mathbf{0}
\end{array}\right] \\
\bar{B}_{i}:=\left[\begin{array}{ll}
B_{i}^{\top} & D_{1 i}^{\top} B_{f i}^{\top}
\end{array}\right] \\
\bar{L}_{0 i}:=\left[\begin{array}{ll}
L_{i}-D_{f i} C_{i} & -C_{f 0 i}
\end{array}\right] \\
\bar{L}_{1 i}:=\left[\begin{array}{ll}
L_{d i}-D_{f i} C_{d i} & -C_{f 1 i}
\end{array}\right] \\
\bar{L}_{2 i}:=\left[\begin{array}{ll}
L_{d i}-D_{f i} C_{d i} & -C_{f 2 i}
\end{array} \bar{L}_{d i}:=\left[\begin{array}{ll}
L_{d i}-D_{f i} C_{d i} & \mathbf{0}
\end{array}\right], \quad D_{i}:=D_{2 i}-D_{f i} D_{1 i} .\right.
\end{array}\right.
$$

By a TTA to the state-delay, the original system (4) is converted into an FI form, which is comprised by a forward subsystem $\left(\mathcal{L}_{d 1}\right)$ with two known constant time-delays and a feedback one $\left(\mathcal{L}_{d 2}\right)$ with delay uncertainties. The idea behind this reformulation is that the stability issue of system (4) can be deemed as a robust performance analysis issue for the forward system $\left(\mathcal{L}_{d 1}\right)$ with constant delays against norm-bounded uncertainties $\Delta_{d}(t)$. Evidently, the stochastic stability of the transformed system (20) indicates the stochastic stability of the original delayed system (4). Especially, the subsequent criterion could be derived, which also offers a possible selection of the scaling matrices $\left\{T_{\eta}, T_{\xi}\right\} \in \mathcal{T}$.

Lemma 4 [20]: For any nonsingular matrix $V$, the operator $\Delta_{d}$ in (20) meets $\left\|V \Delta_{d} V^{-1}\right\|_{\infty} \leq 1$.

Remark 1: According to the model transformation (18), the feedback subsystem $\left(\mathcal{L}_{d 2}\right)$ bears the property that $\left\|V \Delta_{d} V^{-1}\right\|_{\infty} \leq 1$. Then, in view of Lemmas 1 and 3 , if there exists the scaling matrix $\{V, V\} \in \mathcal{T}$ to satisfy $\left\|V G_{d} V^{-1}\right\|_{\infty}<1$, the IO stochastic stability of system (20) is achieved immediately. As a consequence, a new bounded real lemma for the original system in (4) will be proposed subsequently.

\section{B. New Bounded Real Lemma}

Proposition 1: The FES in (4) is SS with an $\mathscr{H}_{\infty}$ performance $\gamma$, if there exist positive-definite symmetric matrices $\left\{P_{i}, Q_{1 i}, Q_{2 i}, Q_{3 i}, R_{1}, R_{2}, Z_{1}, Z_{2}, S\right\} \in \mathbf{R}^{\left(n_{x}+n_{f}\right) \times\left(n_{x}+n_{f}\right)}$, for each mode $i \in \mathcal{I}$, to guarantee the subsequent matrix inequalities

$$
\begin{aligned}
& \Xi_{i}+\operatorname{Sym}\left\{E^{\top} P_{i} \mathscr{A}_{i}\right\}+\mathscr{A}_{i}^{\top} \mathscr{Z} \mathscr{A}_{i}<0 \\
& \mathcal{Q}_{v}:=\sum_{j=1}^{N} \bar{\lambda}_{i j} Q_{v j}-R_{v} \leq 0, \quad v=1,2
\end{aligned}
$$




$$
\mathcal{Q}_{3}:=\sum_{j=1}^{N} \bar{\lambda}_{i j}\left(Q_{2 j}+Q_{3 j}\right)-R_{2} \leq 0
$$

where





and $f_{i}(h)$ refers to the probability density function (PDF) of sojourn-time $h$ at mode $i$.

Proof: First, let $\mathbb{C}\left[-d_{2}, 0\right]$ be the space of continuous functions evolving on $\left[-d_{2}, 0\right]$, and define $\bar{x}_{S}(t):=\bar{x}(t+$ $s), \bar{x}_{s}(t) \in \mathbb{C}\left[-d_{2}, 0\right], s \in\left[-d_{2}, 0\right]$. Thus $\left\{\left(\bar{x}_{s}(t), r(t)\right), t \geq 0\right\}$ could be deemed a semi-Markov process with the initial condition $\left(\left[\phi_{t}^{\top} \mathbf{0}\right]^{\top}, r(0)\right)$. Introduce the subsequent semi-MLK functional for the FES (20)

$$
V\left(\bar{x}_{s}(t), r(t), t\right):=\sum_{m=1}^{4} V_{m}\left(\bar{x}_{s}(t), r(t), t\right)
$$

where

$$
\left\{\begin{aligned}
V_{1}\left(\bar{x}_{s}(t), r(t), t\right):= & \bar{x}^{\top}(t) P(r(t)) \bar{x}(t) \\
V_{2}\left(\bar{x}_{s}(t), r(t), t\right):= & \int_{t-d(t)}^{t} \bar{x}^{\top}(\theta) Q_{3}(r(t)) \bar{x}(\theta) \mathrm{d} \theta \\
& +\sum_{\nu=1}^{2} \int_{t-d_{v}}^{t} \bar{x}^{\top}(\theta) Q_{v}(r(t)) \bar{x}(\theta) \mathrm{d} \theta \\
V_{3}\left(\bar{x}_{s}(t), r(t), t\right):= & \sum_{\nu=1}^{2} \int_{-d_{v}}^{0} \int_{t+\tau}^{t} \bar{x}^{\top}(\theta) R_{\nu} \bar{x}(\theta) \mathrm{d} \theta \mathrm{d} \tau \\
V_{4}\left(\bar{x}_{s}(t), r(t), t\right):= & d_{1} \int_{-d_{1}}^{0} \int_{t+\tau}^{t} \dot{\bar{x}}^{\top}(\theta) Z_{1} \dot{\bar{x}}(\theta) \mathrm{d} \theta \mathrm{d} \tau \\
& +d \int_{-d_{2}}^{-d_{1}} \int_{t+\tau}^{t} \dot{\bar{x}}^{\top}(\theta) Z_{2} \dot{\bar{x}}(\theta) \mathrm{d} \theta \mathrm{d} \tau .
\end{aligned}\right.
$$

Based on the semi-MLK functional defined in (27), together with Definitions 1 and 2 and Lemmas 3 and 4, it can be concluded that the subsequent condition (29) along $\left(\mathcal{L}_{d 1}\right)$ and $\left(\mathcal{L}_{d 2}\right)$ assures that the FES in $(20)$ is SS with an $\mathscr{H}_{\infty}$ performance $\gamma$ in the context of zero initial conditions and nonzero $w(t) \in L_{2}[0, \infty)$

$$
\begin{gathered}
\mathscr{D}\left[V\left(\bar{x}_{s}(t), r(t), t\right)\right]+\mathcal{E}\left\{\xi_{d}^{\top}(t) S \xi_{d}(t)-\eta_{d}^{\top}(t) S \eta_{d}(t)\right\} \\
\quad+\|\bar{z}\|_{\mathcal{E}_{2}}^{2}-\gamma^{2}\|w\|_{2}^{2}<0
\end{gathered}
$$

where $\mathscr{D}$ denotes the infinitesimal generator. According to the definition of $\mathscr{D}$ [25], one arrives at

$$
\begin{gathered}
\mathscr{D}\left[V\left(\bar{x}_{s}(t), r(t), t\right)\right] \\
:=\lim _{\delta \rightarrow 0^{+}} \frac{1}{\delta}\left[\mathcal { E } \left\{V\left(\bar{x}_{s}(t+\delta), r(t+\delta), t+\delta\right) \mid\right.\right. \\
\left.\left.\bar{x}_{s}(t), r(t)=i\right\}-V\left(\bar{x}_{s}(t), r(t), t\right)\right]
\end{gathered}
$$

For each $r(t)=i \in \mathcal{I}$, adopting the law of total probability and conditional expectation provides

$$
\begin{aligned}
& \mathscr{V}_{1}:=\lim _{\delta \rightarrow 0^{+}} \frac{1}{\delta}\left[\mathcal { E } \left\{\sum _ { j = 1 , j \neq i } ^ { N } \operatorname { P r } \left\{r_{n+1}=j, h_{n+1} \leq h+\delta \mid\right.\right.\right. \\
& \left.r_{n}=i, h_{n+1}>h\right\} \\
& \times \bar{x}^{\top}(t+\delta) P_{j} \bar{x}(t+\delta) \\
& +\operatorname{Pr}\left\{r_{n+1}=i, h_{n+1}>h+\delta \mid r_{n}\right. \\
& \left.=i, h_{n+1}>h\right\} \\
& \left.\left.\times \bar{x}^{\top}(t+\delta) P_{i} \bar{x}(t+\delta)\right\}-\bar{x}^{\top}(t) P_{i} \bar{x}(t)\right] \\
& =\lim _{\delta \rightarrow 0^{+}} \frac{1}{\delta}\left[\mathcal { E } \left\{\sum_{j=1, j \neq i}^{N} \frac{\operatorname{Pr}\left\{r_{n+1}=j, r_{n}=i\right\}}{\operatorname{Pr}\left\{r_{n}=i\right\}}\right.\right. \\
& \times \frac{\operatorname{Pr}\left\{h<h_{n+1} \leq h+\delta \mid r_{n+1}=j, r_{n}=i\right\}}{\operatorname{Pr}\left\{h_{n+1}>h \mid r_{n}=i\right\}} \\
& \times \bar{x}^{\top}(t+\delta) P_{j} \bar{x}(t+\delta) \\
& +\frac{\operatorname{Pr}\left\{h_{n+1}>h+\delta \mid r_{n}=i\right\}}{\operatorname{Pr}\left\{h_{n+1}>h \mid r_{n}=i\right\}} \\
& \left.\left.\times \bar{x}^{\top}(t+\delta) P_{i} \bar{x}(t+\delta)\right\}-\bar{x}^{\top}(t) P_{i} \bar{x}(t)\right] \\
& =\lim _{\delta \rightarrow 0^{+}} \frac{1}{\delta}\left[\mathcal { E } \left\{\sum_{j=1, j \neq i}^{N} \frac{q_{i j}\left(G_{i}(h+\delta)-G_{i}(h)\right)}{1-G_{i}(h)}\right.\right. \\
& \times \bar{x}^{\top}(t+\delta) P_{j} \bar{x}(t+\delta) \\
& \left.+\frac{1-G_{i}(h+\delta)}{1-G_{i}(h)} \bar{x}^{\top}(t+\delta) P_{i} \bar{x}(t+\delta)\right\} \\
& \left.-\bar{x}^{\top}(t) P_{i} \bar{x}(t)\right]
\end{aligned}
$$

where $h$ refers to the time elapsed when the system stays at mode $i$ from the last transition; $G_{i}(h)$ represents the cumulative distribution function (CDF) of the sojourn-time when the system stays in mode $i$, and $q_{i j}:=$ $\left[\left(\operatorname{Pr}\left\{r_{n+1}=j, r_{n}=i\right\}\right) /\left(\operatorname{Pr}\left\{r_{n}=i\right\}\right)\right]=\operatorname{Pr}\left\{r_{n+1}=j \mid r_{n}=i\right\}$ denotes the probability intensity of the system transition from 
mode $i$ to mode $j$. Given a small $\delta, \bar{x}(t+\delta)$ could be the first-order approximated as

$$
\bar{x}(t+\delta)=\bar{x}(t)+\delta \dot{\bar{x}}(t)+o(\delta)=\left(\delta \mathscr{A}_{i}+E\right) \varsigma_{1}(t)+o(\delta)
$$

where

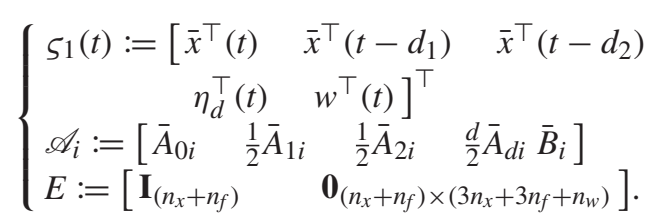

Based on (31)-(33), one obtains

$$
\begin{aligned}
\mathscr{V}_{1}=\lim _{\delta \rightarrow 0^{+}} \frac{1}{\delta}[\mathcal{E}\{ & \sum_{j=1, j \neq i}^{N} \frac{q_{i j}\left(G_{i}(h+\delta)-G_{i}(h)\right)}{1-G_{i}(h)} \\
& \times \varsigma_{1}^{\top}(t)\left(\delta \mathscr{A}_{i}+E\right)^{\top} P_{j}\left(\delta \mathscr{A}_{i}+E\right) \varsigma_{1}(t) \\
& +\frac{1-G_{i}(h+\delta)}{1-G_{i}(h)} \varsigma_{1}^{\top}(t)\left(\delta \mathscr{A}_{i}+E\right)^{\top} P_{i} \\
& \left.\left.\times\left(\delta \mathscr{A}_{i}+E\right) \varsigma_{1}(t)\right\}-\bar{x}^{\top}(t) P_{i} \bar{x}(t)\right] .
\end{aligned}
$$

Considering the condition that $\lim _{\delta \rightarrow 0^{+}}\left[\left(G_{i}(h+\delta)-G_{i}(h)\right) /\right.$ $\left.\left(1-G_{i}(h)\right)\right]=0$, it can be readily derived that

$$
\begin{gathered}
\mathscr{V}_{1}=\varsigma_{1}^{\top}(t)\left[\mathcal { E } \left\{\operatorname { l i m } _ { \delta \rightarrow 0 ^ { + } } \left(\frac{1-G_{i}(h+\delta)}{1-G_{i}(h)} \operatorname{Sym}\left\{E^{\top} P_{i} \mathscr{A}_{i}\right\}\right.\right.\right. \\
\left.\left.\left.+E^{\top} \mathscr{P}_{i} E\right)\right\}\right] \varsigma_{1}(t)
\end{gathered}
$$

where

$$
\begin{aligned}
\mathscr{P}_{i}=\sum_{j=1, j \neq i}^{N} \frac{q_{i j}\left(G_{i}(h+\delta)-G_{i}(h)\right)}{\delta\left(1-G_{i}(h)\right)} P_{j} \\
+\frac{G_{i}(h)-G_{i}(h+\delta)}{\delta\left(1-G_{i}(h)\right)} P_{i} .
\end{aligned}
$$

Utilizing the features of the $\mathrm{CDF}$, we approach to

$$
\begin{aligned}
\lim _{\delta \rightarrow 0^{+}} \frac{1-G_{i}(h+\delta)}{1-G_{i}(h)} & =1 \\
\lim _{\delta \rightarrow 0^{+}} \frac{G_{i}(h+\delta)-G_{i}(h)}{\delta\left(1-G_{i}(h)\right)} & =\lambda_{i}(h)
\end{aligned}
$$

where $\lambda_{i}(h)$ refers to the TR of the system transition from mode $i$.

Define

$$
\lambda_{i j}(h):=q_{i j} \lambda_{i}(h), \quad i \neq j \quad \text { and } \quad \lambda_{i i}(h):=-\sum_{j=1, j \neq i}^{N} \lambda_{i j}(h)
$$

then it follows that:

$$
\mathscr{V}_{1}=\varsigma_{1}^{\top}(t)\left[\operatorname{Sym}\left\{E^{\top} P_{i} \mathscr{A}_{i}\right\}+E^{\top}\left(\sum_{j=1}^{N} \bar{\lambda}_{i j} P_{j}\right) E\right] \varsigma_{1}(t)
$$

where $\bar{\lambda}_{i j}:=\mathcal{E}\left\{\lambda_{i j}(h)\right\}=\int_{0}^{\infty} \lambda_{i j}(h) f_{i}(h) \mathrm{d} h$ with the $\operatorname{PDF} f_{i}(h)$ of sojourn-time $h$ at mode $i$.
With the first term in $V_{2}\left(\bar{x}_{s}(t), r(t), t\right)$, one has

$$
\begin{gathered}
\mathcal{E}\left\{\int_{t+\delta-d(t+\delta)}^{t+\delta} \bar{x}^{\top}(\theta) Q_{3}(r(t+\delta)) \bar{x}(\theta) \mathrm{d} \theta\right\} \\
=\mathcal{E}\left\{\sum_{j=1, j \neq i}^{N} \frac{q_{i j}\left(G_{i}(h+\delta)-G_{i}(h)\right)}{1-G_{i}(h)}\right. \\
\times\left(\delta \bar{x}^{\top}(t) Q_{3 j} \bar{x}(t)+\int_{t-d(t)}^{t} \bar{x}^{\top}(\theta) Q_{3 j} \bar{x}(\theta) \mathrm{d} \theta\right. \\
\quad-\delta(1-\dot{d}(t)) \bar{x}^{\top}(t-d(t)) Q_{3 j} \bar{x}(t-d(t)) \\
\quad+o(\delta))+\frac{1-G_{i}(h+\delta)}{1-G_{i}(h)} \\
\times\left(\delta \bar{x}^{\top}(t) Q_{3 i} \bar{x}(t)+\int_{t-d(t)}^{t} \bar{x}^{\top}(\theta) Q_{3 i} \bar{x}(\theta) \mathrm{d} \theta\right. \\
\quad-\delta(1-\dot{d}(t)) \bar{x}^{\top}(t-d(t)) Q_{3 i} \bar{x}(t-d(t)) \\
+o(\delta))\}
\end{gathered}
$$

Following the similar procedure in (37) for the second term in $V_{2}\left(\bar{x}_{s}(t), r(t), t\right)$, one attains:

$$
\begin{aligned}
& \mathcal{E}\left\{\sum_{\nu=1}^{2} \int_{t+\delta-d_{\nu}}^{t+\delta} \bar{x}^{\top}(\theta) Q_{\nu}(r(t+\delta)) \bar{x}(\theta) \mathrm{d} \theta\right\} \\
& =\sum_{\nu=1}^{2}\left[\sum_{j=1, j \neq i}^{N} \mathcal{E}\left\{\frac{q_{i j}\left(G_{i}(h+\delta)-G_{i}(h)\right)}{1-G_{i}(h)}\right\}\right. \\
& \times\left(\delta \bar{x}^{\top}(t) Q_{v j} \bar{x}(t)+\int_{t-d_{v}}^{t} \bar{x}^{\top}(\theta) Q_{v j} \bar{x}(\theta) \mathrm{d} \theta\right. \\
& \left.-\delta \bar{x}^{\top}\left(t-d_{\nu}\right) Q_{v j} \bar{x}\left(t-d_{v}\right)+o(\delta)\right) \\
& +\mathcal{E}\left\{\frac{1-G_{i}(h+\delta)}{1-G_{i}(h)}\right\} \\
& \times\left(\delta \bar{x}^{\top}(t) Q_{v i} \bar{x}(t)+\int_{t-d_{v}}^{t} \bar{x}^{\top}(\theta) Q_{v i} \bar{x}(\theta) \mathrm{d} \theta\right. \\
& \left.\left.-\delta \bar{x}^{\top}\left(t-d_{v}\right) Q_{v i} \bar{x}\left(t-d_{v}\right)+o(\delta)\right)\right] .
\end{aligned}
$$

According to (30), (37), and (38), one further comes to

$$
\begin{aligned}
\mathscr{V}_{2}=\lim _{\delta \rightarrow 0^{+}} \frac{1}{\delta}\left[\mathcal { E } \left\{V_{2}\left(\bar{x}_{S}(t+\delta), r(t+\delta), t+\delta\right) \mid\right.\right. \\
\left.\left.\bar{x}_{S}(t), r(t)=i\right\}-V_{2}\left(\bar{x}_{s}(t), r(t), t\right)\right] \\
=\lim _{\delta \rightarrow 0^{+}} \frac{1}{\delta}\left\{\sum_{j=1, j \neq i}^{N} \mathcal{E}\left\{\frac{q_{i j}\left(G_{i}(h+\delta)-G_{i}(h)\right)}{1-G_{i}(h)}\right\}\right. \\
\times \int_{t-d(t)}^{t} \bar{x}^{\top}(\theta) Q_{3 j} \bar{x}(\theta) \mathrm{d} \theta
\end{aligned}
$$




$$
\begin{aligned}
& +\mathcal{E}\left\{\frac{1-G_{i}(h+\delta)}{1-G_{i}(h)}\right\} \int_{t-d(t)}^{t} \bar{x}^{\top}(\theta) Q_{3 i} \bar{x}(\theta) \mathrm{d} \theta \\
& +\delta\left(\bar{x}^{\top}(t) Q_{3 i} \bar{x}(t)\right. \\
& \left.\quad-(1-\dot{d}(t)) \bar{x}^{\top}(t-d(t)) Q_{3 i} \bar{x}(t-d(t))\right) \\
& +\sum_{\nu=1}^{2}\left(\sum_{j=1, j \neq i}^{N} \mathcal{E}\left\{\frac{q_{i j}\left(G_{i}(h+\delta)-G_{i}(h)\right)}{1-G_{i}(h)}\right\}\right. \\
& \quad \times \int_{t-d_{\nu}}^{t} \bar{x}^{\top}(\theta) Q_{\nu j} \bar{x}(\theta) \mathrm{d} \theta \\
& +\mathcal{E}^{\prime}\left\{\frac{1-G_{i}(h+\delta)}{1-G_{i}(h)}\right\} \\
& \quad \times \int_{t-d_{\nu}}^{t} \bar{x}^{\top}(\theta) Q_{\nu i} \bar{x}(\theta) \mathrm{d} \theta \\
& +\delta\left(\bar{x}^{\top}(t) Q_{\nu i} \bar{x}(t)-\bar{x}^{\top}\left(t-d_{\nu}\right)\right. \\
& \left.\left.\times Q_{\nu i} \bar{x}\left(t-d_{\nu}\right)\right)\right) \\
& \left.-V_{2}\left(\bar{x}_{s}(t), r(t), t\right)+o(\delta)\right\} .
\end{aligned}
$$

Similarly, it follows that:

$$
\begin{aligned}
\mathscr{V}_{2} \leq & \bar{x}^{\top}(t) Q_{3 i} \bar{x}(t)-(1-\mu) \bar{x}^{\top}(t-d(t)) Q_{3 i} \bar{x}(t-d(t)) \\
& +\int_{t-d(t)}^{t} \bar{x}^{\top}(\theta)\left(\sum_{j=1}^{N} \bar{\lambda}_{i j} Q_{3 j}\right) \bar{x}(\theta) \mathrm{d} \theta \\
& +\sum_{\nu=1}^{2}\left(\bar{x}^{\top}(t) Q_{\nu i} \bar{x}(t)-\bar{x}^{\top}\left(t-d_{v}\right) Q_{v i} \bar{x}\left(t-d_{v}\right)\right) \\
& +\int_{t-d_{1}}^{t} \bar{x}^{\top}(\theta)\left(\sum_{j=1}^{N} \bar{\lambda}_{i j} Q_{1 j}\right) \bar{x}(\theta) \mathrm{d} \theta \\
& +\int_{t-d_{2}}^{t-d(t)} \bar{x}^{\top}(\theta)\left(\sum_{j=1}^{N} \bar{\lambda}_{i j} Q_{2 j}\right) \bar{x}(\theta) \mathrm{d} \theta \\
& +\int_{t-d(t)}^{t} \bar{x}^{\top}(\theta)\left(\sum_{j=1}^{N} \bar{\lambda}_{i j} Q_{2 j}\right) \bar{x}(\theta) \mathrm{d} \theta
\end{aligned}
$$

In addition, we also have

$$
\begin{aligned}
\mathscr{V} / 3= & \sum_{\nu=1}^{2} d_{\nu} \bar{x}^{\top}(t) R_{\nu} \bar{x}(t)-\int_{t-d_{1}}^{t} \bar{x}^{\top}(\theta) R_{1} \bar{x}(\theta) \mathrm{d} \theta \\
& -\int_{t-d(t)}^{t} \bar{x}^{\top}(\theta) R_{2} \bar{x}(\theta) \mathrm{d} \theta-\int_{t-d_{2}}^{t-d(t)} \bar{x}^{\top}(\theta) R_{2} \bar{x}(\theta) \mathrm{d} \theta \\
\mathscr{V}_{4}= & d_{1}^{2} \dot{\bar{x}}^{\top}(t) Z_{1} \dot{\bar{x}}(t)-d_{1} \int_{t-d_{1}}^{t} \dot{\bar{x}}^{\top}(\theta) Z_{1} \dot{\bar{x}}(\theta) \mathrm{d} \theta \\
& +d^{2} \dot{\bar{x}}^{\top}(t) Z_{2} \dot{\bar{x}}(t)-d \int_{t-d(t)}^{t-d_{1}} \dot{\bar{x}}^{\top}(\theta) Z_{2} \dot{\bar{x}}(\theta) \mathrm{d} \theta \\
& -d \int_{t-d_{2}}^{t-d(t)} \dot{\bar{x}}^{\top}(\theta) Z_{2} \dot{\bar{x}}(\theta) \mathrm{d} \theta
\end{aligned}
$$

Utilizing Jensen inequality in [37], we achieve that

$$
\begin{aligned}
\mathscr{V}_{4} \leq & d_{1}^{2} \dot{\bar{x}}^{\top}(t) Z_{1} \dot{\bar{x}}(t)+d^{2} \dot{\bar{x}}^{\top}(t) Z_{2} \dot{\bar{x}}(t) \\
& -\left(\bar{x}(t)-\bar{x}\left(t-d_{1}\right)\right)^{\top} Z_{1}\left(\bar{x}(t)-\bar{x}\left(t-d_{1}\right)\right) \\
& -\sum_{\nu=1}^{2}\left(\bar{x}(t-d(t))-\bar{x}\left(t-d_{\nu}\right)\right)^{\top} Z_{2} \\
& \times\left(\bar{x}(t-d(t))-\bar{x}\left(t-d_{\nu}\right)\right) .
\end{aligned}
$$

Hence, in view of the semi-MLK functional in (27), together with considering (36)-(43), it follows that:

$$
\begin{aligned}
\operatorname{LHS}(29) \leq & S_{1}^{\top}(t)\left[\Xi_{i}+\operatorname{Sym}\left\{E^{\top} P_{i} \mathscr{A}_{i}\right\}+\mathscr{A}_{i}^{\top} \mathscr{Z} \mathscr{A}_{i}\right] \\
& \times \varsigma_{1}(t)+\mathcal{Q}(t)
\end{aligned}
$$

where LHS (29) represents the left-hand side of inequality (29), and

$$
\begin{aligned}
& \mathcal{Q}(t):=\int_{t-d_{1}}^{t} \bar{x}^{\top}(\theta) \mathcal{Q}_{1} \bar{x}(\theta) \mathrm{d} \theta+\int_{t-d_{2}}^{t-d(t)} \bar{x}^{\top}(\theta) \mathcal{Q}_{2} \bar{x}(\theta) \mathrm{d} \theta \\
& +\int_{t-d(t)}^{t} \bar{x}^{\top}(\theta) \mathcal{Q}_{3} \bar{x}(\theta) \mathrm{d} \theta \\
& \mathcal{Q}_{v}:=\sum_{j=1}^{N} \bar{\lambda}_{i j} Q_{v j}-R_{v} \leq 0, v=1,2 \\
& \mathcal{Q}_{3}:=\sum_{j=1}^{N} \bar{\lambda}_{i j}\left(Q_{2 j}+Q_{3 j}\right)-R_{2} \\
& \Xi_{i}:=\Theta_{i}+E^{\top}\left(\sum_{j=1}^{N} \bar{\lambda}_{i j} P_{j}\right) E+\mathscr{L}_{i}^{\top} \mathscr{L}_{i} \\
& \Theta_{i}:=\left[\begin{array}{cccc}
\mathbf{0} & \mathbf{0} & \mathbf{0} & \mathbf{0} \\
-\frac{d}{4}(1-\mu) Q_{3 i} & \mathbf{0} & -\frac{d}{4}(1-\mu) Q_{3 i} & \mathbf{0} \\
-\frac{d}{4}(1-\mu) Q_{3 i} & \mathbf{0} & -\frac{d}{4}(1-\mu) Q_{3 i} & \mathbf{0} \\
\Upsilon_{4} & \mathbf{0} & \Upsilon_{4} & \mathbf{0} \\
* & -\gamma^{2} \mathbf{I} & * & -\gamma^{2} \mathbf{I}
\end{array}\right] \\
& \Upsilon_{1}:=Q_{1 i}+Q_{2 i}+Q_{3 i}+d_{1} R_{1}+d_{2} R_{2}-Z_{1} \\
& \Upsilon_{2}:=-Q_{1 i}-\frac{1}{4}(1-\mu) Q_{3 i}-Z_{1}-\frac{1}{2} Z_{2} \\
& \Upsilon_{3}:=-Q_{2 i}-\frac{1}{4}(1-\mu) Q_{3 i}-\frac{1}{2} Z_{2} \\
& \Upsilon_{4}:=-\frac{d^{2}}{4}(1-\mu) Q_{3 i}-\frac{d^{2}}{2} Z_{2}-S
\end{aligned}
$$



and $\mathscr{A}_{i}, E$, and $\varsigma_{1}(t)$ are defined in (33).

From (22)-(24), we have that LHS (29) $<0$, which renders that the FES (4) is SS with an $\mathscr{H}_{\infty}$ performance $\gamma$. The proof is thus completed.

Remark 2: It is worthy of pointing out that the analysis procedure in Proposition 1 is fundamentally a straightforward utilization of the model transformation and stochastic SSG theorem. By virtue of the semi-MLK functional in (27), together with Lemmas 3 and 4, a semi-MLK format of stochastic SSG condition (29) is presented. Actually, the bounded real lemma can also be proposed by adopting the direct Lyapunov approach, and the result is correspondingly elaborated as follows. 
Corollary 1: For the interconnected system described by $\left(\mathcal{L}_{d 1}\right)$ and $\left(\mathcal{L}_{d 2}\right)$ in $(20)$, if there exists a semi-MLK functional candidate

$$
\begin{aligned}
\bar{V}\left(\bar{x}_{S}(t), r(t), t\right):= & \frac{1}{d} \int_{-d_{2}}^{-d_{1}} \int_{t+\tau}^{t} \xi_{d}^{\top}(\theta) S \xi_{d}(\theta) \mathrm{d} \theta \mathrm{d} \tau \\
& +V\left(\bar{x}_{S}(t), r(t), t\right)
\end{aligned}
$$

where $V\left(\bar{x}_{S}(t), r(t), t\right)$ is defined in (27), satisfying

$$
\mathscr{D}\left[\bar{V}\left(\bar{x}_{S}(t), r(t), t\right)\right]+\|\bar{z}\|_{\mathcal{E}_{2}}^{2}-\gamma^{2}\|w\|_{2}^{2}<0
$$

then the semi-MJLS (4) is SS with an $\mathscr{H}_{\infty}$ performance $\gamma$, in the context of zero initial conditions and nonzero $w(t) \in$ $L_{2}[0, \infty)$.

Proof: By taking the weak infinitesimal generator of $\bar{V}\left(\bar{x}_{S}(t), r(t), t\right)$ along the trajectories of (20) and applying Jensen inequality, we have

$$
\begin{aligned}
\mathscr{D}[ & \left.\bar{V}\left(\bar{x}_{S}(t), r(t), t\right)\right] \\
=\mathscr{D}\left[V\left(\bar{x}_{S}(t), r(t), t\right)\right]+\mathscr{D}\left\{\frac{1}{d} \int_{-d_{2}}^{-d_{1}}\right. & \left.\times \int_{t+\tau}^{t} \xi_{d}^{\top}(\theta) S \xi_{d}(\theta) \mathrm{d} \theta \mathrm{d} \tau\right\} \\
= & \mathscr{D}\left[V\left(\bar{x}_{S}(t), r(t), t\right)\right]+\mathcal{E}\left\{\xi_{d}^{\top}(t) S \xi_{d}(t)\right\} \\
& -\frac{1}{d} \mathcal{E}\left\{\int_{-d_{2}}^{-d_{1}} \xi_{d}^{\top}(t+\tau) S \xi_{d}(t+\tau) \mathrm{d} \tau\right\} \\
\leq & \mathscr{D}\left[V\left(\bar{x}_{s}(t), r(t), t\right)\right]+\mathcal{E}\left\{\xi_{d}^{\top}(t) S \xi_{d}(t)\right\} \\
& -\mathcal{E}\left\{\left[\int_{-d_{2}}^{-d_{1}} \xi_{d}(t+\theta) \mathrm{d} \theta\right]^{\top} S\left[\int_{-d_{2}}^{-d_{1}} \xi_{d}(t+\theta) \mathrm{d} \theta\right]\right\} \\
= & \mathscr{D}\left[V\left(\bar{x}_{S}(t), r(t), t\right)\right]+\mathcal{E}\left\{\xi_{d}^{\top}(t) S \xi_{d}(t)-\eta_{d}^{\top}(t) S \eta_{d}(t)\right\} .
\end{aligned}
$$

It is easy to see that (47) together with (48) is equivalent to the semi-MLK formulation of stochastic SSG condition (29). Performing some similar manipulations as in the proof of Proposition 1, the $\mathscr{H}_{\infty}$ performance analysis criterion can be obtained subsequently. The proof is thus completed.

Notice that there exist coupling terms involved by the Lyapunov matrices and system matrices in condition (22), which bring some obstacle for filter synthesis problem. In [36], a traditional decoupling inequality $-P_{i} Z_{v}^{-1} P_{i} \leq Z_{v}-2 P_{i}, v=$ 1,2 is employed to eliminate the product between the system matrices and Lyapunov variables. In this case, however, notice that the Lyapunov matrices $Z_{1}, Z_{2}$, and $S$ are all constrained by the Lyapunov matrix $P_{i}$, which will lead to much conservativeness. An alternative filter synthesis method is based on the projection lemma [38], which enables one to introduce a set of auxiliary semi-Markovian switching slack variables to separate the Lyapunov variables from the system matrices. It is anticipated that this formulation will reduce the conservativeness of the resulting analysis conditions by means of the incremental flexibility from those Lyapunov variables and additional slack matrices.
For the filter synthesis procedure, we resort to the subsequent projection lemma.

Lemma 5 [38]: Considering matrices $\mathscr{W}=\mathscr{W}^{\top} \in \mathbf{R}^{n \times n}$, $\mathscr{U} \in \mathbf{R}^{k \times n}$, and $\mathscr{V} \in \mathbf{R}^{m \times n}$, the following two statements are equivalent.

1) The linear matrix inequality (LMI) $\mathscr{W}+\mathscr{U}^{\top} \mathscr{X}^{\top} \mathscr{V}+$ $\mathscr{V}^{\top} \mathscr{X} \mathscr{U}<0$ is feasible subject to the variable $\mathscr{X}$.

2) The following conditions hold true:

$$
\begin{cases}\mathscr{U}_{\perp}^{\top} \mathscr{W} \mathscr{U}_{\perp}<0 & \text { if } \mathscr{V}_{\perp}=0, \mathscr{U}_{\perp} \neq 0 \\ \mathscr{V}_{\perp}^{\top} \mathscr{W} \mathscr{V}_{\perp}<0 & \text { if } \mathscr{U}_{\perp}=0, \mathscr{V}_{\perp} \neq 0 \\ \mathscr{U}_{\perp}^{\top} \mathscr{W} \mathscr{U}_{\perp}<0, \mathscr{V}_{\perp}^{\top} \mathscr{W}_{\perp}<0 & \text { if } \mathscr{V}_{\perp} \neq 0, \mathscr{U}_{\perp} \neq 0\end{cases}
$$

where $\mathscr{U}_{\perp}$ and $\mathscr{V}_{\perp}$ stand for the right null spaces of $\mathscr{U}$ and $\mathscr{V}$, respectively.

\section{Memory $\mathscr{H}_{\infty}$ Filtering Design}

In this section, on the basis of projection lemma, the $\mathscr{H}_{\infty}$ filter synthesis will be developed, and the filter parameters can be computed in a convex optimization framework in terms of LMIs.

Theorem 1: Consider the semi-MJLS (1) and filter (3). If there exist positive-definite symmetric matrices $\left\{P_{i}, Q_{1 i}, Q_{2 i}, Q_{3 i}, R_{1}, R_{2}, Z_{1}, Z_{2}, S\right\} \in \mathbf{R}^{\left(n_{x}+n_{f}\right) \times\left(n_{x}+n_{f}\right)}$, and matrices $\left\{X_{1 i}, X_{4 i}, X_{6 i}\right\} \in \mathbf{R}^{n_{x} \times n_{x}},\left\{X_{3 i}, X_{5 i}, X_{7 i}\right\} \in \mathbf{R}^{n_{f} \times n_{x}}$, $X_{8 i} \in \mathbf{R}^{\left(2 n_{x}+2 n_{f}+n_{w}\right) \times n_{x}},\left\{X_{2 i}, \bar{A}_{f 0 i}, \bar{A}_{f 1 i}, \bar{A}_{f 2 i}\right\} \in \mathbf{R}^{n_{f} \times n_{f}}, \bar{B}_{f i} \in$ $\mathbf{R}^{n_{f} \times n_{y}},\left\{C_{f 0 i}, C_{f 1 i}, C_{f 2 i}\right\} \in \mathbf{R}^{n_{z} \times n_{f}}$, and $D_{f i} \in \mathbf{R}^{n_{z} \times n_{y}}$, for each mode $i \in \mathcal{I}$, such that for scalars $\epsilon_{m}, m=1,2,3,4$, the conditions (23), (24) and the following LMIs hold:

$$
\left[\begin{array}{cc}
{\left[\begin{array}{cc}
\mathscr{Z} & P_{i} E \\
* & \Theta_{i}+E^{\top}\left(\sum_{j=1}^{N} \bar{\lambda}_{i j} P_{j}\right) \\
* & *
\end{array}\right]+\operatorname{Sym}\left\{\Phi_{i}\right\}\left[\begin{array}{c}
\mathbf{0} \\
\mathscr{L}_{i}^{\top}
\end{array}\right]} \\
\\
<0, \quad i \in \mathcal{I}
\end{array}\right]
$$

where

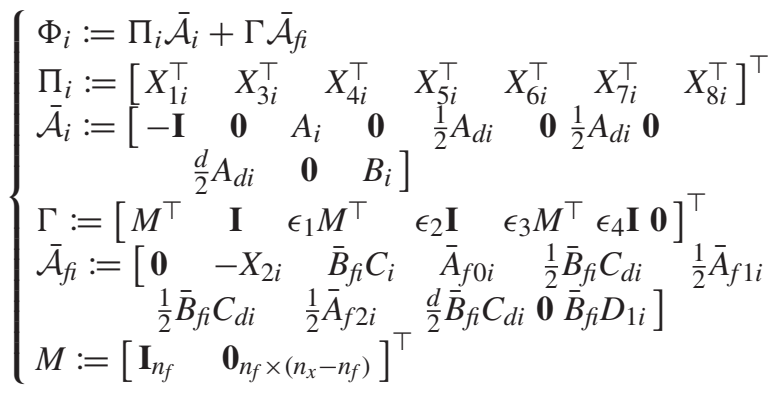

with $\mathscr{Z}, \Theta_{i}, \mathscr{L}_{i}$, and $E$ defined the same as in (26), then the FES in (4) is SS with an $\mathscr{H}_{\infty}$ performance $\gamma$. Specifically, the admissible $n_{f}$-order filter gains can be constructed as

$$
\begin{aligned}
& A_{f 0 i}=X_{2 i}^{-1} \bar{A}_{f 0 i}, \quad A_{f 1 i}=X_{2 i}^{-1} \bar{A}_{f 1 i} \\
& A_{f 2 i}=X_{2 i}^{-1} \bar{A}_{f 2 i}, \quad B_{f i}=X_{2 i}^{-1} \bar{B}_{f i}
\end{aligned}
$$

and $C_{f 0 i}, C_{f 1 i}, C_{f 2 i}$, and $D_{f i}$ can be computed from (49) directly.

Proof: According to Proposition 1, if the condition (22)-(24) hold, then the FES (4) is SS with an $\mathscr{H}_{\infty}$ performance index. For filter design purpose, the 
projection lemma (Lemma 5) will be first utilized to make a decoupling between the system matrices and Lyapunov variables. To this end, we reformulate the inequality (22) as

$$
\left[\begin{array}{c}
\mathscr{A}_{i} \\
\mathbf{I}_{\left(4 n_{x}+4 n_{f}+n_{w}\right)}
\end{array}\right]^{\top}\left[\begin{array}{cc}
\mathscr{Z} & P_{i} E \\
E^{\top} P_{i} & \Xi_{i}
\end{array}\right]\left[\begin{array}{c}
\mathscr{A}_{i} \\
\mathbf{I}_{\left(4 n_{x}+4 n_{f}+n_{w}\right)}
\end{array}\right]<0, \quad i \in \mathcal{I}
$$

where $\Xi_{i}, \mathscr{A}_{i}, \mathscr{Z}$, and $E$ are shown in (26).

Explicit null space computation leads to

$$
\left[\begin{array}{ll}
-\mathbf{I}_{\left(n_{x}+n_{f}\right)} & \mathscr{A}_{i}
\end{array}\right]_{\perp}=\left[\begin{array}{c}
\mathscr{A}_{i} \\
\mathbf{I}_{\left(4 n_{x}+4 n_{f}+n_{w}\right)}
\end{array}\right] .
$$

Utilizing projection lemma, we have the subsequent inequality inferring to (52)

$$
\left[\begin{array}{cc}
\mathscr{Z} & P_{i} E \\
E^{\top} P_{i} & \Xi_{i}
\end{array}\right]+\operatorname{Sym}\left\{\mathscr{X}_{i}\left[-\mathbf{I}_{\left(n_{x}+n_{f}\right)} \quad \mathscr{A}_{i}\right]\right\}<0, \quad i \in \mathcal{I}
$$

where $\mathscr{X}_{i} \in \mathbf{R}^{\left(5 n_{x}+5 n_{f}+n_{w}\right) \times\left(n_{x}+n_{f}\right)}$ is the multiplier.

Now, it follows from (53) that the Lyapunov variables have been separated from the system matrices. Therefore, in the sequel, the main purpose is to convexify the multiplications between the system matrices and the free matrices.

For simplicity in the filter design procedure, we first prescribe the slack variables as



$$
\begin{aligned}
& \mathcal{X}_{1 i}:=\left[\begin{array}{cc}
X_{1 i} & M X_{2} \\
X_{3 i} & X_{4}
\end{array}\right], \quad M:=\left[\begin{array}{c}
\mathbf{I}_{n_{f}} \\
\mathbf{0}_{\left(n_{x}-n_{f}\right) \times n_{f}}
\end{array}\right], \quad i \in \mathcal{I}
\end{aligned}
$$

where $\varepsilon_{1}$ and $\varepsilon_{2}$ are two scalar parameters, and $X_{1 i} \in \mathbf{R}^{n_{x} \times n_{x}}$, $X_{3 i} \in \mathbf{R}^{n_{f} \times n_{x}}$, and $\left\{X_{2}, X_{4}\right\} \in \mathbf{R}^{n_{f} \times n_{f}}$. Then, to linearize the matrix inequality, similar to [36], performing a congruent transformation to

$$
\left[\begin{array}{cc}
X_{1 i}+X_{1 i}^{\top} & M X_{2}+X_{3 i}^{\top} \\
* & X_{4}+X_{4}^{\top^{\prime}}
\end{array}\right]
$$

by $\operatorname{diag}\left\{\mathbf{I}_{n_{x}}, X_{2} X_{4}^{-1}\right\}$ provides

$$
\begin{aligned}
& {\left[\begin{array}{cc}
X_{1 i}+X_{1 i}^{\top} & M X_{2} X_{4}^{-\top} X_{2}^{\top}+X_{3 i}^{\top} X_{4}^{-\top} X_{2}^{\top} \\
* & \underline{\overline{X_{2} X_{4}^{-\top} X_{2}^{\top}}}+X_{2} X_{4}^{-1} X_{2}^{\top}
\end{array}\right]} \\
& :=\left[\begin{array}{cc}
X_{1 i}+X_{1 i}^{\top} & M \overline{\bar{X}}_{2}+\bar{X}_{3 i}^{\top} \\
* & \underline{\overline{\bar{X}_{2}}+\bar{X}_{2}^{\top}}
\end{array}\right] .
\end{aligned}
$$

Therefore, we can further assign the matrix $\mathcal{X}_{1 i}$ with the following form:

$$
\mathcal{X}_{1 i}=\left[\begin{array}{cc}
X_{1 i} & M X_{2 i} \\
X_{3 i} & X_{2 i}
\end{array}\right], \quad i \in \mathcal{I}
$$

Notice that this formulation enables the matrix variable $X_{2 i}$ to be implicitly included into the filter gain variables $A_{f 0 i}, A_{f 1 i}$, $A_{f 2 i}$, and $B_{f i}$ by introducing

$$
\begin{aligned}
& \bar{A}_{f 0 i}:=X_{2 i} A_{f 0 i}, \quad \bar{A}_{f 1 i}:=X_{2 i} A_{f 1 i} \\
& \bar{A}_{f 2 i}:=X_{2 i} A_{f 2 i}, \quad \bar{B}_{f i}:=X_{2 i} B_{f i}
\end{aligned}
$$

which allows all the slack variables to be set as semiMarkovian switching.
Moreover, it is noted that the filter gains are not included in the first row of the system matrices $\bar{A}_{0 i}, \bar{A}_{1 i}, \bar{A}_{2 i}, \bar{A}_{d i}$, and $\bar{B}_{i}$ in (21), such that for further conservativeness reduction of design, we can choose the free variables

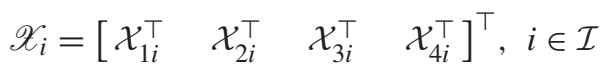

where

$$
\begin{aligned}
& \mathcal{X}_{1 i}:=\left[\begin{array}{cc}
X_{1 i} & M X_{2 i} \\
X_{3 i} & X_{2 i}
\end{array}\right], \quad \mathcal{X}_{2 i}:=\left[\begin{array}{cc}
X_{4 i} & \epsilon_{1} M X_{2 i} \\
X_{5 i} & \epsilon_{2} X_{2 i}
\end{array}\right] \\
& \mathcal{X}_{3 i}:=\left[\begin{array}{cc}
X_{6 i} & \epsilon_{3} M X_{2 i} \\
X_{7 i} & \epsilon_{4} X_{2 i}
\end{array}\right], \quad \mathcal{X}_{4 i}:=\left[\begin{array}{ll}
X_{8 i} & \mathbf{0}
\end{array}\right]
\end{aligned}
$$

with $\left\{X_{1 i}, X_{4 i}, X_{6 i}\right\} \in \mathbf{R}^{n_{x} \times n_{x}}, X_{2 i} \in \mathbf{R}^{n_{f} \times n_{f}},\left\{X_{3 i}, X_{5 i}, X_{7 i}\right\} \in$ $\mathbf{R}^{n_{f} \times n_{x}}, X_{8 i} \in \mathbf{R}^{\left(2 n_{x}+2 n_{f}+n_{w}\right) \times n_{x}}$, and $\epsilon_{1}, \epsilon_{2}, \epsilon_{3}$, and $\epsilon_{4}$ are scalar parameters.

Then, with the substitution of matrix $\mathscr{X}_{i}$ defined in (59) into (53) and application of Schur complement, together with consideration of (58), it is obvious that (53) leads to (49).

Additionally, the conditions in (49) indicate that $-X_{2 i}-$ $X_{2 i}^{\top}<0$, which states that $X_{2 i}$ is nonsingular. Then, the filter parameters can be calculated by (51). The proof is therefore completed.

Remark 3: By invoking projection lemma and introducing a set of auxiliary multipliers $\mathscr{X}_{i}$, Theorem 1 presents a delaydependent condition on memory filter synthesis problem for continuous-time semi-MJLSs in (1) with time-varying delay. Notice that this decoupling characteristic enables one to adopt more slack variables to deal with the product between the system matrices and Lyapunov variables. It is also worth mentioning that this characteristic is quite of usefulness for the filter design of semi-MJLSs since more flexible dimensions in the solution space of LMI-based sufficient conditions, directly result in reducing the conservativeness of the solutions.

Remark 4: Notice that the filter synthesis criteria derived in Theorem 1 are related with the derivative of time-varying delay with $\dot{d}(t) \leq \mu<\infty$. Nevertheless, it could also be easily generalized to the case of delay-derivative-independent results by setting $Q_{1 i}=\mathbf{0}, i \in \mathcal{I}$ in Theorem 1 for the filter design of delayed semi-MJLSs.

\section{Simulation Studies}

Consider a continuous-time semi-MJLS with time-delay (1), which is with three modes and system parameters are shown in (61), as shown at the bottom of the next page. The switches among the three subsystems are governed by a semi-Markov chain, whose TR matrix is characterized with

$$
\begin{aligned}
\Lambda(h) & =\left[\begin{array}{ccc}
\lambda_{11}(h) & \lambda_{12}(h) & \lambda_{13}(h) \\
\lambda_{21}(h) & \lambda_{22}(h) & \lambda_{23}(h) \\
\lambda_{31}(h) & \lambda_{32}(h) & \lambda_{33}(h)
\end{array}\right] \\
= & {\left[\begin{array}{ccc}
-2 h & h & h \\
0.5 h & -h & 0.5 h \\
\frac{2}{9} h & \frac{2}{9} h & -\frac{4}{9} h
\end{array}\right] }
\end{aligned}
$$

By carefully considering the properties of Weibull distribution, it is easy to conclude that the TR functions in (62) could be approximately explained as that the sojourn-time follows the Weibull distribution with PDF $f(h)=\left(\left[v_{2}\right] /\left[v_{1}^{\nu_{2}}\right]\right) h^{\nu_{2}-1}$ 
TABLE I

Minimum $\mathscr{H}_{\infty}$ Performance for Different CASES By Memory/Memoryless Filter With $d_{1}=0.1$ AND $d_{2}=0.5$

\begin{tabular}{|c|c|c|c|c|c|c|c|c|c|c|c|c|}
\hline \multirow{2}{*}{ Filters } & \multicolumn{3}{|c|}{$\mu=0.2$} & \multicolumn{3}{|c|}{$\mu=0.5$} & \multicolumn{3}{|c|}{$\mu=0.9$} & \multicolumn{3}{|c|}{ unknown $\mu$} \\
\hline & $n_{f}=3$ & $n_{f}=2$ & $n_{f}=1$ & $n_{f}=3$ & $n_{f}=2$ & $n_{f}=1$ & $n_{f}=3$ & $n_{f}=2$ & $n_{f}=1$ & $n_{f}=3$ & $n_{f}=2$ & $n_{f}=1$ \\
\hline Case-I & 0.9411 & 0.9821 & 1.2247 & 0.9457 & 0.9896 & 1.2283 & 0.9528 & 0.9920 & 1.2314 & 0.9602 & 0.9996 & 1.2343 \\
\hline Case-II & 1.1552 & 1.2374 & 1.4443 & 1.1578 & 1.2403 & 1.4486 & 1.1589 & 1.2436 & 1.4502 & 1.1603 & 1.2483 & 1.4523 \\
\hline
\end{tabular}

Case-I: memory filter (3)

Case-II: memoryless filter (3) with $A_{f 1 i}=A_{f 2 i}=\mathbf{0}, C_{f 1 i}=C_{f 2 i}=\mathbf{0}, i \in \mathcal{I}$

$\exp \left[-\left(h / \nu_{1}\right)^{\tau}\right], h \geq 0$. Especially, when $i=1$, the TR expression $h$ could be described as a Weibull distribution with the scale parameter $v_{1}=1$ and the shape parameter $v_{2}=2$, i.e., $f_{1}(h)=2 h \mathrm{e}^{-h^{2}}$; when $i=2$, the TR expression $0.5 h$ could be modeled by the Weibull distribution with $\nu_{1}=2$ and $v_{2}=2$, that is $f_{2}(h)=0.5 h \mathrm{e}^{-0.25 h^{2}}$; when $i=3$, the TR expression $2 / 9 h$ could be described by the Weibull distribution with $v_{1}=3$ and $v_{2}=2$, i.e., $f_{3}(h)=2 / 9 h \mathrm{e}^{-1 / 9 h^{2}}$. Correspondingly, we can compute the mathematical expectation of TR $\lambda_{12}(h)$ as $\mathcal{E}\left\{\lambda_{12}(h)\right\}=\int_{0}^{\infty} h f_{1}(h) \mathrm{d} h=\int_{0}^{\infty} 2 h^{2} \mathrm{e}^{-h^{2}} \mathrm{~d} h=0.8862 \mathrm{~s}$. With some similar algebraic manipulations to calculate the mathematical expectation of the other TRs, we have

$$
\mathcal{E}\{\Lambda(h)\}=\left[\begin{array}{ccc}
-1.7722 & 0.8861 & 0.8861 \\
1.7726 & -3.5452 & 1.7726 \\
2.6587 & 2.6587 & -5.3174
\end{array}\right]
$$

The purpose is to synthesize a mode-dependent filter (3) such that the resulting FES (4) is SS with an $\mathscr{H}_{\infty}$ performance index. Notice when $A_{f 1 i}=A_{f 2 i}=\mathbf{0}$ and $C_{f 1 i}=C_{f 2 i}=\mathbf{0}$, $i \in \mathscr{I}$, the memory filter (3) reduces to a memoryless one. To this end, suppose that the lower and upper bounds of time-delay are $d_{1}=0.1$ and $d_{2}=0.5$, respectively. It has been checked that the delay-dependent approaches developed in [38] and [39] yield no feasible solutions. Coming back to Theorem 1 with $\epsilon_{1}=\epsilon_{2}=\epsilon_{3}=\epsilon_{4}=1$, offers the feasible solution, and a detailed comparison of the attained minimum $\mathscr{H}_{\infty}$ performance indices $\gamma_{\min }$ by the memory/memoryless filters with various delay-derivatives is summarized in Table I, where unknown $\mu$ refers to the corresponding delay-derivative-independent filter synthesis results, as illustrated in Remark 4.

By inspection of Table I, it can be readily found that a memory filter can generally achieve better performances than a memoryless one. Thus, the superiority of the memory filters over memoryless ones is apparent.

In particular, with the time-varying delay $0.1 \leq d(t) \leq 0.5$, with $\dot{d}(t) \leq 0.9$, adopting Theorem 1 leads to the feasible solutions of $\gamma_{\min }=0.9528$ for the full-order filter, $\gamma_{\min }=0.9920$

$\left[\begin{array}{c|c|c}A_{1} & A_{d 1} & B_{1} \\ \hline C_{1} & C_{d 1} & D_{11} \\ \hline L_{1} & L_{d 1} & D_{21}\end{array}\right]=\left[\begin{array}{ccc|ccc|c}-3 & 1 & 0 & -0.2 & 0.1 & 0.6 & 1 \\ 0.3 & -2.5 & 1 & 0.5 & -1 & -0.8 & 0 \\ -0.1 & 0.3 & -3.8 & 0 & 1 & -2.5 & 1 \\ \hline 0.8 & 0.3 & 0 & 0.2 & -0.3 & -0.6 & 0.2 \\ \hline 0.5 & -0.1 & v 1 & 0 & 0.1 & 0.2 & 0.1\end{array}\right]$

$\left[\begin{array}{c|c|c}A_{2} & A_{d 2} & B_{2} \\ \hline C_{2} & C_{d 2} & D_{12} \\ \hline L_{2} & L_{d 2} & D_{22}\end{array}\right]=\left[\begin{array}{ccc|ccc|c}-3.5 & 0.8 & 0.5 & -0.9 & -1.3 & 0.2 & -0.6 \\ 0.6 & -3.3 & 5 & -0.7 & -2.1 & 0.5 & 0.5 \\ -0.1 & 1 & -2 & -0.8 & -1.0 & 0.6 & 0 \\ \hline-0.5 & 0.2 & 0.3 & 0 & -0.6 & 0.2 & 0.5 \\ \hline 0 & 1 & 0.6 & 0.2 & 0.1 & 0 & -0.1\end{array}\right]$

$\left[\begin{array}{c|c|c}A_{3} & A_{d 3} & B_{3} \\ \hline C_{3} & C_{d 3} & D_{13} \\ \hline L_{3} & L_{d 3} & D_{23}\end{array}\right]=\left[\begin{array}{ccc|ccc|c}-2.5 & 0.5 & -0.1 & 0 & -0.3 & 0.6 & 0.5 \\ 0.1 & -3.5 & 0.3 & 0.1 & 0.5 & 0 & 0.4 \\ -0.1 & 1 & -2 & -0.6 & 1 & -0.8 & 1 \\ \hline-0.6 & 0.7 & 0.4 & 0.7 & -0.3 & 0.5 & -0.3 \\ \hline-0.3 & 0.1 & 0.8 & 0.3 & -0.1 & 0.2 & 0.3\end{array}\right]$

$\digamma_{1}=\left[\begin{array}{cc|cc|cc|c}-2.7941 & 1.1076 & -0.9766 & -0.8235 & -2.3480 & 0.3462 & 2.1303 \\ -1.4656 & -1.8234 & 0.8900 & -0.9985 & 0.2211 & -1.9011 & -2.6566 \\ \hline-0.9200 & -0.1199 & 0.5293 & -1.3924 & 0.0668 & -0.1087 & -0.0414\end{array}\right]$
$\digamma_{2}=\left[\begin{array}{cc|cc|cc|c}-1.1488 & 0.1395 & -2.2046 & -0.0092 & 0.1015 & 0.1563 & -3.0438 \\ 0.6134 & -0.2557 & -1.3052 & -1.5337 & -1.2844 & 0.1656 & -3.6531 \\ \hline-0.1069 & -0.0590 & -0.0882 & -0.3929 & 0.0620 & -0.3675 & -0.5381\end{array}\right]$
$\digamma_{3}=\left[\begin{array}{ll|ll|c|c|c}0.1408 & 0.0772 & -0.4404 & -0.1811 & 0.0885 & 0.1507 & -0.1409 \\ 0.2183 & 0.1177 & -0.6019 & -0.3475 & -0.3001 & 0.0116 & -1.4676 \\ \hline 0.1744 & 0.0969 & 0.0409 & 0.0291 & 0.0286 & -0.0552 & 0.6605\end{array}\right]$




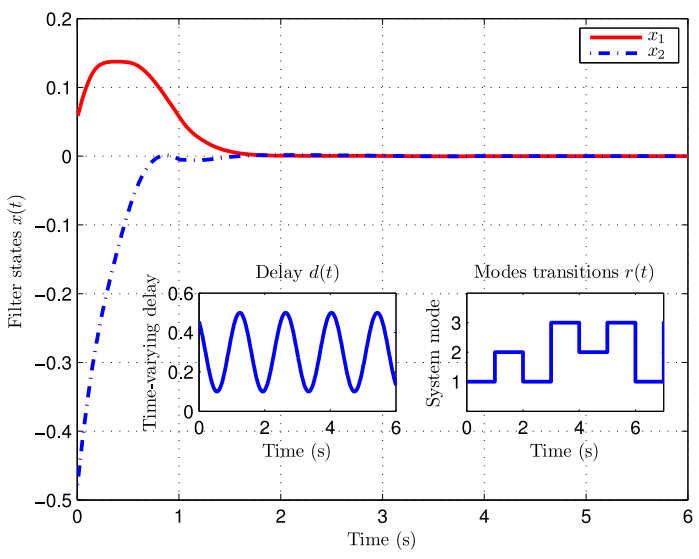

Fig. 3. State responses of the reduced-order filter (second-order filter).

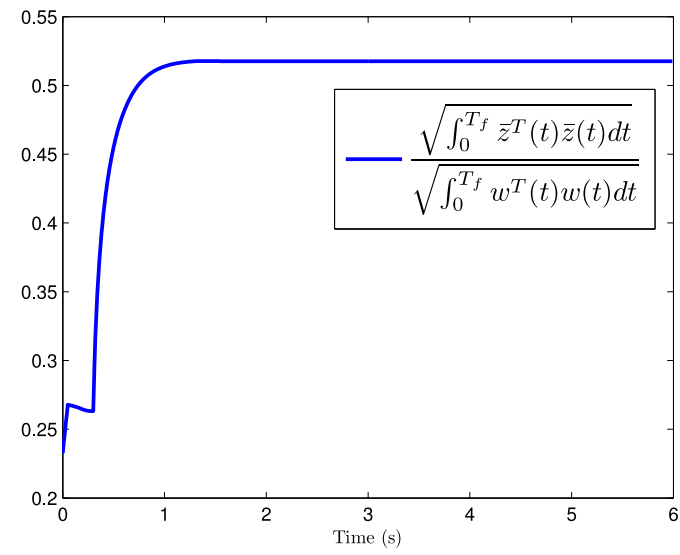

Fig. 4. Response of the ratio $\sqrt{\int_{0}^{T_{f}} \bar{z}^{\top}(t) \bar{z}(t) \mathrm{d} t} / \sqrt{\int_{0}^{T_{f}} w^{\top}(t) w(t) \mathrm{d} t}$.

for the second-order filter and $\gamma_{\min }=1.2314$ for the first-order filter, respectively. Meantime, the filter gains for the secondorder filter are given in (63), as shown at the bottom of the previous page, where

$$
\digamma_{i}:=\left[\begin{array}{l|l|l|l}
A_{f 0 i} & A_{f 1 i} & A_{f 2 i} & B_{f i} \\
\hline C_{f 0 i} & C_{f 1 i} & C_{f 2 i} & D_{f i}
\end{array}\right], i=1,2,3 .
$$

The filter parameters for the other cases are omitted for brevity.

To further validate the efficacy of the presented results, some simulation studies are exhibited with the initial condition $x(t)=\left[\begin{array}{lll}5 \mathrm{e}^{2 t} \sin (3.14 t) & 0 & 5 \mathrm{e}^{0.5 t} \sin (0.32 t)\end{array}\right]^{\top}, t \in$ $[-0.5,0]$. Fig. 3 shows the state estimation responses in the context of time-varying delay $d(t)=0.3+0.2 \sin (4.5 t)$, and the external disturbance $w(t)=10 \mathrm{e}^{-3 t} \sin (0.015 t)$, and Fig. 4 shows the time response of the ratio $\sqrt{\int_{0}^{T_{f}} \bar{z}^{\top}(t) \bar{z}(t) \mathrm{d} t} / \sqrt{\int_{0}^{T_{f}} w^{\top}(t) w(t) \mathrm{d} t}$ with zero initial condition. It can be observed from Fig. 4 that the time response of ratio $\sqrt{\int_{0}^{T_{f}} \bar{z}^{\top}(t) \bar{z}(t) \mathrm{d} t} / \sqrt{\int_{0}^{T_{f}} w^{\top}(t) w(t) \mathrm{d} t}$ is less than 0.6, which is evidently less than the prescribed disturbance attenuation level 0.9920 , and thus the designed memory filter achieves satisfactory performance.

\section{CONCLUSION}

In this paper, the delay-dependent $\mathscr{H}_{\infty}$ memory filtering issue for continuous-time semi-MJLSs with time-delay has been addressed via an IO approach. A TTA method has been first employed to reformulate the original system into an FI formulation, which consists of a forward subsystem with two constant time-delays and a feedback one with delay uncertainties. Then, on the basis of a semi-MLK format of stochastic SSG condition combined with projection lemma, the $\mathscr{H}_{\infty}$ filter synthesis has been presented. It has been shown that with a linearization technique, the desired full- and reducedorder filter parameters can be calculated in a unified frame. Simulation studies have been carried out to reveal the effectiveness and advantage of the developed method. It is noted that the combination of the IO approach and some newly developed techniques, i.e., Wirtinger-based integral inequality and free-weighting matrix method [42]-[47], could further reduce the analysis conservativeness of semi-MJLSs with time-delay, which would motivate us for investigation as future works.

\section{REFERENCES}

[1] M. Mariton, Jump Linear Systems in Automatic Control. New York, NY, USA: M. Dekker, 1990.

[2] X. Feng, K. A. Loparo, Y. Ji, and H. J. Chizeck, "Stochastic stability properties of jump linear systems," IEEE Trans. Autom. Control, vol. 37, no. 1, pp. 38-52, Jan. 1992.

[3] C. L. P. Chen, Y.-J. Liu, and G.-X. Wen, "Fuzzy neural network-based adaptive control for a class of uncertain nonlinear stochastic systems," IEEE Trans. Cybern., vol. 44, no. 5, pp. 583-593, May 2014.

[4] C. L. P. Chen, G.-X. Wen, Y.-J. Liu, and F.-Y. Wang, "Adaptive consensus control for a class of nonlinear multiagent time-delay systems using neural networks," IEEE Trans. Neural Netw. Learn. Syst., vol. 25, no. 6, pp. 1217-1226, Jun. 2014.

[5] C. L. P. Chen, J. Wang, C.-H. Wang, and L. Chen, "A new learning algorithm for a fully connected neuro-fuzzy inference system," IEEE Trans. Neural Netw. Learn. Syst., vol. 25, no. 10, pp. 1741-1757, Oct. 2014.

[6] Z. Wang, D. W. C. Ho, and X. Liu, "A note on the robust stability of uncertain stochastic fuzzy systems with time-delays," IEEE Trans. Syst., Man, Cybern. A, Syst., Humans, vol. 34, no. 4, pp. 570-576, Jul. 2004.

[7] Z. Wang, Y. Liu, and X. Liu, “ $\mathscr{H}_{\infty}$ filtering for uncertain stochastic timedelay systems with sector-bounded nonlinearities," Automatica, vol. 44, no. 5, pp. 1268-1277, May 2008.

[8] Y. Liu, Z. Wang, Y. Yuan, and W. Liu, "Event-triggered partial-nodesbased state estimation for delayed complex networks with bounded distributed delays," IEEE Trans. Syst., Man, Cybern., Syst., to be published, doi: 10.1109/TSMC.2017.2720121.

[9] Z. Wang, Y. Liu, and X. Liu, "Exponential stabilization of a class of stochastic system with Markovian jump parameters and modedependent mixed time-delays," IEEE Trans. Autom. Control, vol. 55, no. 7, pp. 1656-1662, Jul. 2010.

[10] J. Liu, L. Zha, J. Cao, and S. Fei, "Hybrid-driven-based stabilisation for networked control systems," IET Control Theory Appl., vol. 10, no. 17 pp. 2279-2285, Nov. 2016.

[11] S. Ding and S. Li, "Second-order sliding mode controller design subject to mismatched term," Automatica, vol. 77, pp. 388-392, Mar. 2017.

[12] Y. Wei, J. Qiu, and H. R. Karimi, "Reliable output feedback control of discrete-time fuzzy affine systems with actuator faults," IEEE Trans. Circuits Syst. I, Reg. Papers, vol. 64, no. 1, pp. 170-181, Jan. 2017.

[13] S. Ding, A. Levant, and S. Li, "Simple homogeneous sliding-mode controller," Automatica, vol. 67, pp. 22-32, May 2016.

[14] Y. Wei, J. Qiu, H. R. Karimi, and M. Wang, "Model approximation for two-dimensional Markovian jump systems with state-delays and imperfect mode information," Multidimensional Syst. Signal Process., vol. 26, no. 3, pp. 575-597, Jul. 2015.

[15] Y. Wei, J. Qiu, H. K. Lam, and L. Wu, "Approaches to T-S fuzzy-affinemodel-based reliable output feedback control for nonlinear Itô stochastic systems," IEEE Trans. Fuzzy Syst., vol. 25, no. 3, pp. 569-583, Jun. 2017 
[16] R. Samidurai, R. Manivannan, C. K. Ahn, and H. R. Karimi, "New criteria for stability of generalized neural networks including Markov jump parameters and additive time delays," IEEE Trans. Syst., Man, Cybern., Syst., to be published, doi: 10.1109/TSMC.2016.2609147.

[17] Y. Wei, J. Qiu, H. R. Karimi, and M. Wang, "Quantized $\mathscr{H}_{\infty}$ filtering for continuous-time Markovian jump systems with deficient mode information," Asian J. Control, vol. 17, no. 5, pp. 1914-1923, Sep. 2015.

[18] J. Liu, J. Cao, Z. Wu, and Q. Qi, "State estimation for complex systems with randomly occurring nonlinearities and randomly missing measurements," Int. J. Syst. Sci., vol. 45, no. 7, pp. 1364-1374, Jul. 2014.

[19] Y. Wei, J. Qiu, H. R. Karimi, and M. Wang, " $\mathscr{H}_{\infty}$ model reduction for continuous-time Markovian jump systems with incomplete statistics of mode information," Int. J. Syst. Sci., vol. 45, no. 7, pp. 1496-1507, Jul. 2014.

[20] Y. Wei, J. Qiu, H. R. Karimi, and M. Wang, "New results on $\mathscr{H}_{\infty}$ dynamic output feedback control for Markovian jump systems with time-varying delay and defective mode information," Opt. Control Appl. Methods, vol. 35, no. 6, pp. 656-675, Nov./Dec. 2014.

[21] L. Campo, P. Mookerjee, and Y. Bar-Shalom, "State estimation for systems with sojourn-time-dependent Markov model switching," IEEE Trans. Autom. Control, vol. 36, no. 2, pp. 238-243, Feb. 1991.

[22] H. Zhang, W. Ni, X. Li, and Y. Yang, "Modeling the heterogeneous duration of user interest in time-dependent recommendation: A hidden semi-Markov approach," IEEE Trans. Syst., Man, Cybern., Syst., to be published, doi: 10.1109/TSMC.2016.2599705.

[23] L. Zhang, T. Yang, P. Shi, and M. Liu, "Stability and stabilization of a class of discrete-time fuzzy systems with semi-Markov stochastic uncertainties," IEEE Trans. Syst., Man, Cybern., Syst., vol. 46, no. 12, pp. 1642-1653, Dec. 2016.

[24] E. Shmerling and K. J. Hochberg, "Stability of stochastic jumpparameter semi-Markov linear systems of differential equations," Stochastics Int. J. Probab. Stochastic Processes Formerly Stochastics Stochastics Rep., vol. 80, no. 6, pp. 513-518, Oct. 2008.

[25] J. Huang and Y. Shi, "Stochastic stability and robust stabilization of semi-Markov jump linear systems," Int. J. Robust Nonlin. Control, vol. 23, no. 18, pp. 2028-2043, Dec. 2013.

[26] Y. Foucher, E. Mathieu, P. Saint-Pierre, J. F. Durand, and J. Daurès, "A semi-Markov model based on generalized Weibull distribution with an illustration for HIV disease," Biometrical J., vol. 47, no. 6, pp. 825-833, 2005.

[27] B. Chen, C. Lin, X. Liu, and K. Liu, "Observer-based adaptive fuzzy control for a class of nonlinear delayed systems," IEEE Trans. Syst., Man, Cybern., Syst., vol. 46, no. 1, pp. 27-36, Jan. 2016.

[28] Y. Wei, J. Qiu, P. Shi, and H.-K. Lam, "A new design of $H$-infinity piecewise filtering for discrete-time nonlinear time-varying delay systems via T-S fuzzy affine models," IEEE Trans. Syst., Man, Cybern., Syst., vol. 47, no. 8, pp. 2034-2047, Aug. 2017.

[29] J. Song, S. He, Z. Ding, and F. Liu, "A new iterative algorithm for solving $\mathscr{H}_{\infty}$ control problem of continuous-time Markovian jumping linear systems based on online implementation," Int. J. Robust Nonlin. Control, vol. 26, no. 17, pp. 3737-3754, Nov. 2016.

[30] K. Gu, V. L. Kharitonov, and J. Chen, Stability of Time-Delay Systems. Boston, MA, USA: Birkhäuser, 2003.

[31] J. Zhang, C. R. Knopse, and P. Tsiotras, "Stability of time-delay systems: Equivalence between Lyapunov and scaled small-gain conditions," IEEE Trans. Autom. Control, vol. 46, no. 3, pp. 482-486, Mar. 2001.

[32] E. Fridman and U. Shaked, "Input-output approach to stability and $L_{2}$ gain analysis of systems with time-varying delays," Syst. Control Lett., vol. 55, no. 12, pp. 1041-1053, Dec. 2006.

[33] S. He, J. Song, and F. Liu, "Robust finite-time bounded controller design of time-delay conic nonlinear systems using sliding mode control strategy," IEEE Trans. Syst., Man, Cybern., Syst., to be published, doi: 10.1109/TSMC.2017.2695483.

[34] Y. Wei, J. Qiu, and H.-K. Lam, "A novel approach to reliable output feedback control of fuzzy-affine systems with timedelays and sensor faults," IEEE Trans. Fuzzy Syst., to be published, doi: 10.1109/TFUZZ.2016.2633323.

[35] X. Kai, C. Wei, and L. Liu, "Robust extended Kalman filtering for nonlinear systems with stochastic uncertainties," IEEE Trans. Syst., Man, Cybern. A, Syst., Humans, vol. 40, no. 2, pp. 399-405, Mar. 2010.
[36] H. Gao, J. Lam, L. Xie, and C. Wang, "New approach to mixed $\mathscr{H}_{2} / \mathscr{H}_{\infty}$ filtering for polytopic discrete-time systems," IEEE Trans. Signal Process., vol. 53, no. 8, pp. 3183-3192, Aug. 2005.

[37] H.-N. Wu, J.-W. Wang, and P. Shi, "A delay decomposition approach to $\mathscr{L}_{2}-\mathscr{L}_{\infty}$ filter design for stochastic systems with time-varying delay," Automatica, vol. 47, no. 7, pp. 1482-1488, Jul. 2011.

[38] J. Qiu, G. Feng, and J. Yang, "A new design of delay-dependent robust $\mathscr{H}_{\infty}$ filtering for continuous-time polytopic systems with time-varying delay," Int. J. Robust Nonlin. Control, vol. 20, no. 3, pp. 346-365, Feb. 2010.

[39] B. Zhang, W. X. Zheng, and S. Xu, "On robust $\mathscr{H}_{\infty}$ filtering of uncertain Markovian jump time-delay systems," Int. J. Adapt. Control Signal Process., vol. 26, no. 2, pp. 138-157, Feb. 2012.

[40] Y. He, Q.-G. Wang, and C. Lin, "An improved $\mathscr{H}_{\infty}$ filter design for systems with time-varying interval delay," IEEE Trans. Circuits Syst. II, Exp. Briefs, vol. 53, no. 11, pp. 1235-1239, Nov. 2006.

[41] L. Frezzatto, M. J. Lacerda, R. C. L. F. Oliveira, and P. L. D. Peres, "Robust $\mathscr{H}_{2}$ and $\mathscr{H}_{\infty}$ memory filter design for linear uncertain discretetime delay systems," Signal Process., vol. 117, pp. 322-332, Dec. 2015.

[42] A. Seuret and F. Gouaisbaut, "Wirtinger-based integral inequality: Application to time-delay systems," Automatica, vol. 49, no. 9, pp. 2860-2866, Sep. 2013.

[43] C.-K. Zhang, Y. He, L. Jiang, M. Wu, and H.-B. Zeng, "Stability analysis of systems with time-varying delay via relaxed integral inequalities," Syst. Control Lett., vol. 92, pp. 52-61, Jun. 2016.

[44] Y. Chen, S. Fei, and Y. Li, "Robust stabilization for uncertain saturated time-delay systems: A distributed-delay-dependent polytopic approach," IEEE Trans. Autom. Control, vol. 62, no. 7, pp. 3455-3460, Jul. 2017.

[45] Y. Zhang, "Stochastic stability of discrete-time Markovian jump delay neural networks with impulses and incomplete information on transition probability," Neural Netw., vol. 46, pp. 276-282, Oct. 2013.

[46] Y. Zhang, "Stability of discrete-time Markovian jump delay systems with delayed impulses and partly unknown transition probabilities," Nonlin. Dyn., vol. 75, nos. 1-2, pp. 101-111, Jan. 2014.

[47] Y. Zhang and C. Wang, "Robust stochastic stability of uncertain discretetime impulsive Markovian jump delay systems with multiplicative noises," Int. J. Syst. Sci., vol. 46, no. 12, pp. 2210-2220, Dec. 2015. 